\title{
A DPOC na Área Metropolitana do Porto e o efeito das ondas de calor e de frio na enfermidade
}

\section{COPD at the Porto Metropolitan Area and the effect of heat and cold waves on this disease}

Rafael Brito Silveira, LabClima, Departamento de Geociências, Universidade Federal de Santa Catarina, Brasil, rafael.brito@posgrad.ufsc.br

(1) https://orcid.org/0000-0002-7062-3540

Maikon Passos Amilton Alves, LabClima, Departamento de Geociências, Universidade Federal de Santa Catarina, Brasil, maiconpassos@gmail.com

(1) https://orcid.org/0000-0002-3328-2265

Carlos Miguel Sousa, Instituto Geofísico, Universidade do Porto, Portugal, miguelsousa83@gmail.com

(1) https://orcid.org/0000-0002-0924-0866

Paula Gonçalves, Instituto Geofísico, Universidade do Porto, Portugal, paulagoncalves1993@gmail.com

(1) https://orcid.org/0000-0003-3740-8336

Ana Monteiro, CEGOT, Departamento Geografia, Universidade do Porto, Portugal, anamonteirosousa@gmail.com

(1) https://orcid.org/0000-0002-3392-2664

Alberto Elvino Franke, LabClima, Departamento de Geociências, Universidade Federal de Santa Catarina, Brasil, alberto.franke@ufsc.br

(1) https://orcid.org/0000-0002-0222-4652

Resumo: Caracterizou-se a doença pulmonar obstrutiva crónica (DPOC) e os possíveis aspectos socioeconómicos associados à enfermidade e avaliaram-se os efeitos que as ondas de calor (OdC) e as ondas de frio (OdF) podem ter no aumento dos internamentos hospitalares nas pessoas residentes na Área Metropolitana do Porto (AMP) entre 2000 e 2015. Os efeitos das OdC e das OdF nas admissões hospitalares por DPOC foram investigados por meio da análise de regressão linear múltipla, com nível de significância de $\alpha=5 \%$. A análise estatística e cartográfica da distribuição geográfica dos internamentos hospitalares por DPOC, por grupos etários, por taxa de analfabetismo e por taxa de desemprego, à escala de freguesia, permitiu explicar os efeitos das OdC e das OdF no agravamento desta enfermidade. Do ponto de vista da caracterização das admissões por DPOC na AMP, concluiuse que, em geral, esta doença está associada às freguesias com condições socioeconómicas mais precárias e com a população mais envelhecida. A intensidade e a duração, de acordo com o modelo utilizado, explicam o aumento nas taxas de admissões por DPOC durante os eventos de OdC e OdF. Todavia, a duração contribuiu mais nos eventos de calor e a intensidade nos eventos de frio. A severidade média foi o único parâmetro que não compôs o modelo.

Palavras-chave: DPOC; Área Metropolitana do Porto; Onda de calor; Onda de frio.

Abstract: Chronic obstructive pulmonary disease (COPD) and the possible socioeconomic aspects associated with the disease were characterized and the effects that heat waves (HW) and cold waves (CW) can have on the increase of hospitalizations in people living in the Porto Metropolitan Area (PMA) between 2000 and 2015 were evaluated. The effects of HW and CW on hospital admissions for COPD were investigated by multiple linear regression analysis, with a significance level of $\alpha=5 \%$. Statistical and cartographic analysis of the geographic distribution of hospitalizations for COPD in the age groups, illiteracy rate and unemployment rate at the parish scale, explained the effects of $\mathrm{HW}$ and $\mathrm{CW}$ on the aggravation of this disease. From the point of view of the characterization of COPD admissions in PMA, it was concluded that, in general, this disease is associated with parishes with poorer socioeconomic 
conditions and the older population. Intensity and duration, according to the model used, explain the increase in COPD admission rates during the HW and CW events. However, duration contributed more in heat events and intensity in cold events. Average severity was the only parameter that did not make up the model.

Keywords: COPD; Porto Metropolitan Area; Heat waves; Cold waves.

\section{Introdução}

A busca de relações entre clima e saúde tem sido uma preocupação constante dos seres humanos e são inúmeros os exemplos, desde a Antiguidade Clássica. Diferentes pesquisas em décadas passadas exibem as percepções e as evidências vinculadas aos impactos ambientais, incluindo os climáticos, sobre as condições de saúde das pessoas (e.g. Rogers, 1925; Kimble, 1938; Sorre, 1951; Miller, 1953; Maunder, 1970; Lacaz et al., 1972; Peixoto, 1975). Kimble (1938, p. 214), por exemplo, no livro Geography in the Middle Ages, relata como alguns elementos climáticos, durante a Idade Média, podiam gerar danos para a saúde humana ou carregar a pestilência sob sua incumbência.

Os estudos que investigam os possíveis impactos dos elementos climáticos na saúde humana envolvem análises de diversas patologias, entre elas, aquelas ligadas ao sistema respiratório (e.g. Langford, Bentham, 1995; Ballester et al., 2003; Yusuf et al., 2007; Oluleye, Akinbobola, 2010; Nair et al., 2010; Monteiro et al., 2013a; Huang, Barnett, 2014; Gasparrini et al., 2015; Numminen et al., 2015; Zhao et al., 2019). Dentre os elementos do clima integrados nos estudos de bioclimatologia, a temperatura do ar é um dos mais estudados e, muitas vezes, relacionado com as doenças do aparelho respiratório, inclusive em Portugal (Almeida et al., 2010; Lin, Zacharek, 2012; Bárbara et al., 2013; Monteiro et al., 2013a; Antunes et al., 2016).

O considerável impacto que os extremos de temperatura causam na saúde pública, principalmente nas enfermidades referentes ao aparelho respiratório, torna essa temática uma questão pertinente para a sociedade (O'neill et al., 2005; Sarfaty, Abouzaid, 2009), neste caso, portuguesa.

Dentro das doenças respiratórias que podem ser impactadas pelos elementos do clima está a doença pulmonar obstrutiva crónica (DPOC). Em 2016, segundo a World Health Organization (WHO, 2018), a DPOC foi a terceira enfermidade que mais causou mortes em todo o mundo, seguida pelas infecções respiratórias inferiores e, atrás apenas, da doença cardíaca isquémica e do acidente vascular cerebral. De acordo com o Sistema Nacional de Saúde (SNS) de Portugal, o número de utentes inscritos nos cuidados primários diagnosticados com DPOC, entre 2011 e 2017, para todo o país, foi de 136.958 no total. Deste montante, a área Norte, onde se encontra a Área Metropolitana do Porto (AMP), registrou 55.402 utentes ( 40\%) (Ministério da Saúde, 2017, 2018).

A DPOC é um termo usual utilizado para retratar as doenças pulmonares progressivas, compreendendo enfisema, bronquite crónica e asma não reversível. 
Esta enfermidade é marcada pelo aumento da falta de ar. A DPOC é uma doença evolutiva e, nos dias de hoje, ainda sem cura, todavia, com o diagnóstico e o tratamento preciso é possível controlá-la (COPD Foundation, s/d). Segundo Durmaz et al. (2015), a DPOC é uma patologia crónica altamente prevalente nas vias aéreas, com grande impacto pessoal e social, associada à alta morbidade e mortalidade e está projetada para ser a terceira causa mais comum de morte no mundo até 2030 (WHO, 2008). A patologia tem um considerável índice de mortalidade em todo o planeta e os fatores de risco, de forma geral, incluem fatores genéticos, a asma, o tabagismo, a poluição atmosférica, a idade e o sexo (Seo e Lee, 2019). O tabagismo é apontado por diversas pesquisas como o principal fator de risco ligado à DPOC (Campos, Lemos, 2009; Pessôa, Pessôa, 2009). A DPOC, sendo uma doença respiratória, é agravada, dentre outras causas, pela temperatura do ar, pela umidade e também pela falta de um ambiente arejado (Monteiro et al., 2012). Como exemplo, afere-se que, em Portugal, os custos com um doente com DPOC em estado de exacerbação grave possa chegar até 2.250,00 € (Ministério da Saúde, 2017b, p. 13).

Apesar de a AMP contar com 17 concelhos, neste estudo excluíram-se os de Arouca $(A R C)$ e Vale de Cambra (VCB) (Figura 1a), pelo fato de estarem situados em subtipos climáticos bastante distintos (Figura 1b). De acordo com Monteiro et al. (2012), a AMP possui quatro subtipos climáticos, com a maior parte do território enquadrada no Atlântico e no Atlântico ameno. Uma vez que a proposta é investigar os possíveis impactos das ondas de calor e de frio na DPOC, a exclusão dos dois concelhos justifica-se, pois estão inseridos, maioritariamente, em subtipos climáticos com características distintas (Atlântico de transição fresco e de Altitude), o que poderia afetar a análise. A população da AMP no Censo de 2011, com a exclusão dos dois concelhos supracitados, era de 1.714.301 habitantes, distribuída em quase $1.600 \mathrm{~km}^{2}$, o que representava $16,2 \%$ do total da população de Portugal (INE, 2011).

Daveau $(1995$, p. 47,48$)$ afirma que no verão os tipos de tempo em Portugal são determinados pelas condições anticiclónicas e pela existência de sistemas de baixa pressão na parte do interior da Península Ibérica e na África setentrional. Ao longo do litoral, as águas costeiras do oceano Atlântico encontram-se arrefecidas pela elevação recorrente de água profunda, criando nestas áreas uma camada de ar fresco e húmido. De maneira geral, as demais áreas do país são influenciadas pela massa de ar ibérica, de características quente e seca, especialmente no interior (Daveau, 1995, p. 48). Na estação invernal, as frentes polares ditam a dinâmica atmosférica e seus respectivos tipos de tempo. Além disto, nesta mesma estação, os ventos oceânicos de oeste penetram vastamente no país, homogeneizando a temperatura do ar até se depararem com os contrafortes, evidenciando a influência do relevo (Daveau, 1995, p. 47, 48). Portanto, as frentes polares e o Anticiclone dos Açores são os sistemas que, basicamente, definem as condições de tempo em Portugal continental (Daveau, 1995).

Esta pesquisa tem dois objetivos principais convergentes: 1) caracterizar a DPOC na AMP e os possíveis aspectos socioeconómicos associados à enfermidade e; 2) avaliar 
os efeitos das OdC e OdF nas admissões hospitalares por DPOC na AMP entre 2000 e 2015.

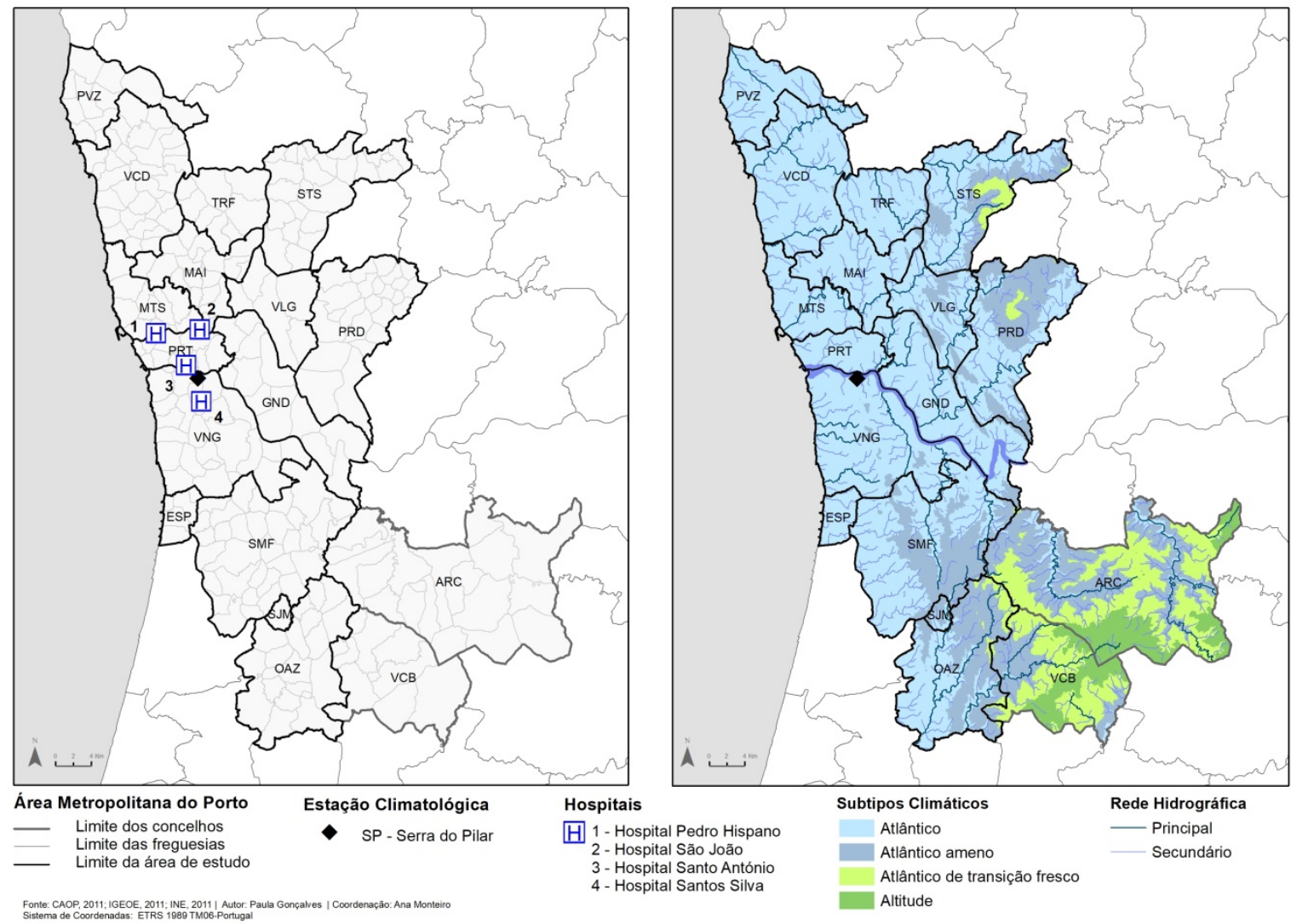

Figura 1: Localização da AMP, da estação climatológica Serra do Pilar e dos hospitais públicos incluídos no estudo (à esquerda). Subtipos climáticos da AMP (à direita). Adaptado de Monteiro et al. (2012).

A influência dos eventos de temperatura extrema nas enfermidades do foro respiratório tem merecido a atenção de cada vez mais investigadores. No que diz respeito às ondas de calor $(\mathrm{OdC})$ e às ondas de frio $(\mathrm{OdF})$, os exemplos só no novo milênio são vários (e.g. Huynen et al., 2001; Anderson, Bell, 2009; D'lppoliti et al., 2010; Barnett et al., 2012; Monteiro et al., 2013a; Silveira et al., 2018).

Todavia, os critérios de classificação do que deve ser considerado um valor térmico extremo são múltiplos. Neste contributo aproveitar-se-á o resultado da metodologia de detecção de OdC e OdF aplicada em Alves et al. (no prelo) para o período 19702017, a partir das estações climatológicas da Serra do Pilar e de Pedras Rubras.

\section{Materiais e métodos}

Os casos de DPOC foram selecionados utilizando o grupo de diagnóstico homogéneo (GDH) 88, proveniente da base de dados fornecida pela Administração Central dos Serviços de Saúde (ACSS) com os registros diários de admissão no período 20002015. Os registros da patologia são apenas analisados neste período por conta da 
disponibilidade dos dados, pois carecem de validação por parte dos órgãos competentes. As admissões foram provenientes dos seguintes hospitais: Pedro Hispano, em Matosinhos; São João e Santo António, no Porto; e Santos Silva, em Vila Nova de Gaia (Figura 1a).

Conforme supracitado, as OdC e as OdF foram extraídas de Alves et al. (no prelo). A técnica empregada pelos autores identifica as $\mathrm{OdC}(\mathrm{OdF})$ com base em limites diários que consideram o percentil 90 (10) da $T_{\text {máx }}\left(T_{\text {minn }}\right)$, centrado numa janela de 15 dias (sete dias antes, o dia em questão e os sete dias posteriores). Alves et al. (no prelo) obtiveram uma amostragem de até 720 observações para o cálculo do limiar climatológico diário (P90 e P10), entre 1970 e 2017 (48 anos). Todavia, aqui se utilizou apenas os eventos detectados no período 2000-2015. Para se considerar uma onda a duração mínima deve ser de três dias consecutivos com $T_{\text {máx }}>$ P90 (OdC) e $T_{\min }<$ P10 (OdF) com base no limiar diário posto. Os autores, além da frequência mensal e anual, analisaram outros três parâmetros, sendo eles: duração $(D)$, intensidade (Int) e severidade média $(\mathrm{Sm})$. Os possíveis impactos destes eventos foram analisados a partir dos três parâmetros citados.

Sabe-se que o critério para considerar uma OdC (OdF), preliminarmente, foi criado pela Organização Meteorológica Mundial (OMM), que considera uma OdC (OdF) um período de no mínimo seis dias consecutivos em que a $T_{\text {máx }}\left(T_{\min }\right)$ esteja $5^{\circ} \mathrm{C}$ acima (abaixo) da média do período de referência (WMO, 2015). A partir da criação do termo pela OMM o mesmo banalizou-se e inúmeras pesquisas passaram a atribuir tal nomenclatura para estes eventos. Mas, o método estabelecido pela OMM é muito rigoroso (Alves et al., 2017) e praticamente não detecta OdC e OdF. Ainda, sabe-se que os eventos extremos de temperatura com durações menores do que o recomendado pela OMM também podem ser lesivos em diversos setores da sociedade, inclusive na saúde. Por isto, assim como outras (e.g. Theoharatos et al., 2010; Monteiro et al., 2013b; Geirinhas et al., 2018), a presente pesquisa utiliza um método de detecção diferente e ainda assim atribui a nomenclatura OdC e OdF.

É importante explicar a definição dos três parâmetros que serão aqui analisados, portanto, a duração $(D)$ refere-se ao número de dias em que a $T_{\text {máx }}>$ P90 ou em que a $T_{\min }<\mathrm{P} 10$, ocorre durante, no mínimo, três dias consecutivos. A média da temperatura durante o evento é calculada e chamada de intensidade (Int). Assim, a intensidade da OdC é dada por $\operatorname{Int}(\operatorname{OdC})=1 / \mathrm{D}\left(\sum_{\mathrm{i}}^{\mathrm{D}}\left(\mathrm{T}_{\text {máx }}\right)_{\mathrm{i}}\right)$ e a da OdF é dada por $\operatorname{Int}(\mathrm{OdF})=1 / \mathrm{D}\left(\sum_{\mathrm{i}}^{\mathrm{D}}\left(\mathrm{T}_{\text {mín }}\right)_{\mathrm{i}}\right)$. Já a severidade é determinada por $\mathrm{S}_{\mathrm{OdC}}=\sum_{\mathrm{i}}^{\mathrm{D}}\left(\mid \mathrm{T}_{\text {máx }}-\right.$ $\mathrm{P} 90 \mid)_{\mathrm{i}}$ e por $\mathrm{S}_{\mathrm{OdF}}=\sum_{\mathrm{i}}^{\mathrm{D}}\left(\left|\mathrm{T}_{\text {mín }}-\mathrm{P} 10\right|\right)_{\mathrm{i}}$. Entretanto, aqui será aplicada a severidade média $(S m)$, que é dada por $S m(O d C)=S_{O d C} / D$ para OdC e $S m(O d F)=S_{O d F} / D$ para OdF (Bitencourt et al., no prelo).

Com o intuito de realizar as análises estatísticas entre OdC e OdF junto aos dados da DPOC, as admissões foram transformadas em taxa $(x 100.000)$, uma vez que a população da AMP não é estanque e se modifica ao longo dos anos. Para tal, os dados populacionais dos concelhos foram extraídos do Censo de 2011 e das 
projeções (INE, 2011; PORDATA, s/d). O cálculo da taxa de admissões diária é dado por:

$$
T x \cdot a d m .=\frac{n^{0} \text { de admissões } \times 100.000}{\text { População anual }}
$$

Posto que os dados de admissões da DPOC são diários e que as OdC e as OdF são eventos, foi necessário estabelecer um período de influência dos eventos extremos sob a enfermidade. Algumas pesquisas divergem quanto ao período de influência da temperatura do ar nas admissões. Monteiro et al. (2013a) evidenciam que, para o Porto, a desfasagem é de até duas semanas (14 dias) entre a ocorrência dos eventos de frio extremo e a DPOC. Ferrari et al. (2012) ao estudarem, dentre outros elementos, a influência da temperatura do ar na DPOC, para a região da Bavária (Alemanha), testaram um lag de até sete dias. Diaz et al. (2005) observaram uma média de intervalo de oito dias entre os impactos gerados pelos dias exepcionalmente frios e a ocorrência de mortes por doenças respiratórias na população idosa. Todavia, para a localidade de Vadu na Índia, Ingole et al. (2017), pesquisando sobre a associação entre fatores socioambientais e mortalidades por causas não acidentais relacionadas ao calor e ao frio, concluíram que as maiores associações entre temperatura do ar e mortalidade durante o inverno ocorreram com um gap de 0 a 13 dias. Nota-se como a desfasagem entre os eventos atmosféricos e os problemas de saúde possuem variações temporais, de local para local. Por conta disto optou-se por testar estatisticamente a maior associação entre os parâmetros das OdC e das OdF com as admissões por DPOC no local de estudo. A técnica utilizada para verificar a desfasagem (gap) máxima foi a correlação de Spearman $\left(r_{s}\right)$, uma vez que os dados não apresentaram distribuição normal a partir do teste de Shapiro-Wilk $(W)(\alpha=5 \%)$. Utilizando-se os dados da estação climatológica da Serra do Pilar, foram calculadas as correlações entre a temperatura máxima ( $\left.T_{\text {máx }}\right)$, a temperatura mínima $\left(T_{\text {mín }}\right)$ e as admissões por DPOC, em periodicidade diária, no período 2000-2015. Constata-se que, para ambas as variáveis, o gap que demonstra a maior correlação foi o de 14 dias (Tabela I).

Tabela I: Teste de gap a partir da correlação de Spearman $\left(r_{s}\right)$ - Serra do Pilar - 2000 a 2015

\begin{tabular}{lcccccccc}
\hline Gap (dias) & 0 & 1 & 2 & 3 & 4 & 5 & 6 & 7 \\
\hline$T_{\operatorname{máx}}$ & $-0,313^{*}$ & $-0,314^{*}$ & $-0,324^{*}$ & $-0,320^{*}$ & $-0,324^{*}$ & $-0,330^{*}$ & $-0,331^{*}$ & $-0,338^{*}$ \\
$T_{\min }$ & $-0,324^{*}$ & $-0,318^{*}$ & $-0,320^{*}$ & $-0,323^{*}$ & $-0,326^{*}$ & $-0,330^{*}$ & $-0,328^{*}$ & $-0,333^{*}$ \\
\hline Gap (dias) & 8 & 9 & 10 & 11 & 12 & 13 & 14 & 15 \\
\hline$T_{\operatorname{máx}}$ & $-0,343^{*}$ & $-0,347^{*}$ & $-0,346^{*}$ & $-0,350^{*}$ & $-0,345^{*}$ & $-0,350^{*}$ & $\mathbf{- 0 , 3 5 2 ^ { * }}$ & $-0,345^{*}$ \\
$T_{\min }$ & $-0,335^{*}$ & $-0,338^{*}$ & $-0,337^{*}$ & $-0,345^{*}$ & $-0,346^{*}$ & $-0,349^{*}$ & $-\mathbf{0 , 3 5 0 ^ { * }}$ & $-0,342^{*}$ \\
\hline
\end{tabular}

$\left(^{*}\right)$ Significativo ao nível de $\alpha=1 \%(p<0,01)$.

Nota-se que todas as correlações $\left(r_{s}\right)$ foram negativas/inversas, isto é, quando a temperatura do ar decai as internações aumentam. Assim, atribuíram-se todas as admissões registradas até o $14^{\circ}$ dia posterior ao último dia de uma onda. Este gap de 14 dias fez com que ocorressem nas OdC uma sobreposição de até cinco eventos e, 
no caso das OdF, de até três. O período de duas semanas também é um intervalo suficiente para que as pessoas procurem as unidades de saúde dentro do período epidemiológico.

Para avaliar os impactos dos parâmetros relativos às $\mathrm{OdC}$ e às $\mathrm{OdF}$ nas admissões por DPOC, aplicou-se o teste de regressão linear múltipla (RLM). A RLM é utilizada em análises em que mais do que uma variável independente $(x)$ pode influenciar na variável dependente $(y)$ (Rogerson, 2012, p. 225). Com $n$ variáveis explicativas independentes, a equação da RLM é:

$$
\hat{y}=a+b_{1} x_{1}+b_{2} x_{2}+\cdots+b_{n} x_{n}
$$

Onde: $\hat{y}$ é o valor predito da variável dependente; a é o coeficiente de interseção (intercepto); $b$ é o coeficiente das variáveis investigadas $e ; x$ é o valor da variável independente.

Primeiramente, de forma separada, testou-se a correlação entre os parâmetros das ondas e a taxa de admissão para verificar quais destes $(D$, Int e $S m)$ iriam compor a análise de RLM. A correlação utilizada também foi a de Sperman $\left(r_{s}\right)$, pois os dados não apresentaram distribuição normal, verificadas a partir do teste de Shapiro-Wilk (W) $(\alpha=5 \%)$. As correlações entre os parâmetros das OdC e a taxa de admissão por DPOC demonstram que apenas a $S m$ não foi significativa $(0,88)$. Todavia, todos os parâmetros das OdF evidenciam significância estatística para as correlações (Tabela II). Logo, apenas a Sm na análise das OdC não foi investigada na análise de RLM.

Tabela II: Correlação de Spearman $\left(r_{s}\right)$ - Tx. de admissão Vs. parâmetros das OdC e OdF - Serra do Pilar - 2000 a 2015

\begin{tabular}{lcrrcrrr}
\hline \multicolumn{1}{c}{ OdC } & \multicolumn{1}{c}{$n$} & \multicolumn{1}{c}{$r_{s}$} & \multicolumn{1}{c}{$p$-valor } & OdF & \multicolumn{1}{c}{$n$} & \multicolumn{1}{c}{$r_{s}$} & $p$-valor \\
\hline Duração & 71 & 0,50 & $<0,05$ & Duração & 23 & 0,62 & $<0,05$ \\
Intensidade & 71 & $-0,54$ & $<0,05$ & Intensidade & 23 & $-0,83$ & $<0,05$ \\
Severidade & 71 & $-0,01$ & 0,88 & Severidade & 23 & 0,45 & $<0,05$ \\
\hline
\end{tabular}

Todos os testes estatísticos foram realizados nos softwares PAST 3.21 (Hammer et al., 2001) e Statistica, versão 10. Os mapas apresentados no estudo foram confeccionados utilizando o ArcGIS 10.4.1.

O mapa da taxa de admissão por DPOC em cada freguesia (Figura 2) utilizou os registros totais ao longo dos 16 anos avaliados e para relativizá-lo, o número de casos foi transformado em taxas. Sendo assim calculadas:

$$
\text { Tx. }=\frac{n^{\mathrm{o}} \text { de admissões } x 10.000}{\text { População da freguesia }}
$$

Obs.: a taxa neste caso foi calculada por 10.000 para não exacerbar os intervalos de classe no mapa.

Já os mapas por freguesia referentes aos grupos etários (\%), a taxa de analfabetismo e a taxa de desemprego (Figuras 3 e 4) foram feitos utilizando os dados do Censo de 
2011 (INE, 2011). Todos os intervalos estabelecidos nos mapas foram determinados pelo método natural breaks.

\section{Resultados e discussão}

\subsection{Aspectos socioeconómicos atrelados à $D P O C$ na $A M P$}

Com o intuito de mostrar a distribuição geográfica das características socioeconómicas das admissões por DPOC na AMP ao longo dos anos estudados, os registros da enfermidade e de alguns aspectos socioeconómicos foram cartografados.

Nota-se que, de modo geral, as maiores taxas de admissão por DPOC estão nas freguesias situadas no centro da AMP e em suas cercanias. Os destaques são as freguesias tocantes aos concelhos de: Porto (PRT), Matosinhos (MTS), Valongo (VLG), Maia (MAl), Gondomar (GND) e Espinho (ESP), tais sítios são aqueles que apresentam em pelo menos uma freguesia, taxas de admissão nas classes mais críticas: $151-200$ e 201-358. Vila Nova de Gaia (VNG), embora possua a maior população da AMP com 302.295 habitantes (INE, 2011), não está na classe de maior destaque (Figura 2).

Supõe-se que a proveniência dos registros de DPOC utilizados na presente pesquisa possa contribuir para essa distribuição espacial da patologia, pois são oriundos de quatro hospitais (Figuras 1 e 2) que estão, justamente, situados na zona central da AMP, dentro dos concelhos que, via de regra, apresentaram as maiores taxas. Outro aspecto que pode ter influência nesta distribuição, mesmo sendo relativizado, é a população total dos concelhos, uma vez que, além de serem as localidades mais próximas aos hospitais, são também as mais populosas da AMP e, consequentemente, os maiores aglomerados urbanos. As demais freguesias dos concelhos periféricos do sul, do norte e mais a leste, apresentaram taxas mais baixas de admissão por DPOC. Isto pode ter explicação pela falta de dados procedentes de hospitais mais próximos de tais sítios. Além disto, a maior distância entre as freguesias e as unidades de saúde avaliadas pode ter contribuído para estes resultados, pela maior dificuldade de deslocamento e acesso (Figura 2).

De acordo com o Censo de 2011 (INE, 2011), excluindo-se os concelhos de Arouca (ARC) e Vale de Cambra (VCB), a população distribuída em grupos etários estava assim disposta: 257.308 (15\%) jovens (0 a 14 anos); 1.181 .078 (68,9\%) adultos (15 a 64 anos) e; 275.915 (16,1\%) idosos ( $\geq 65$ anos). Segundo Inagaki et al. (2008), a Organização Mundial da Saúde estabelece que são consideradas idosas, nos países desenvolvidos, as pessoas com 65 anos ou mais. 


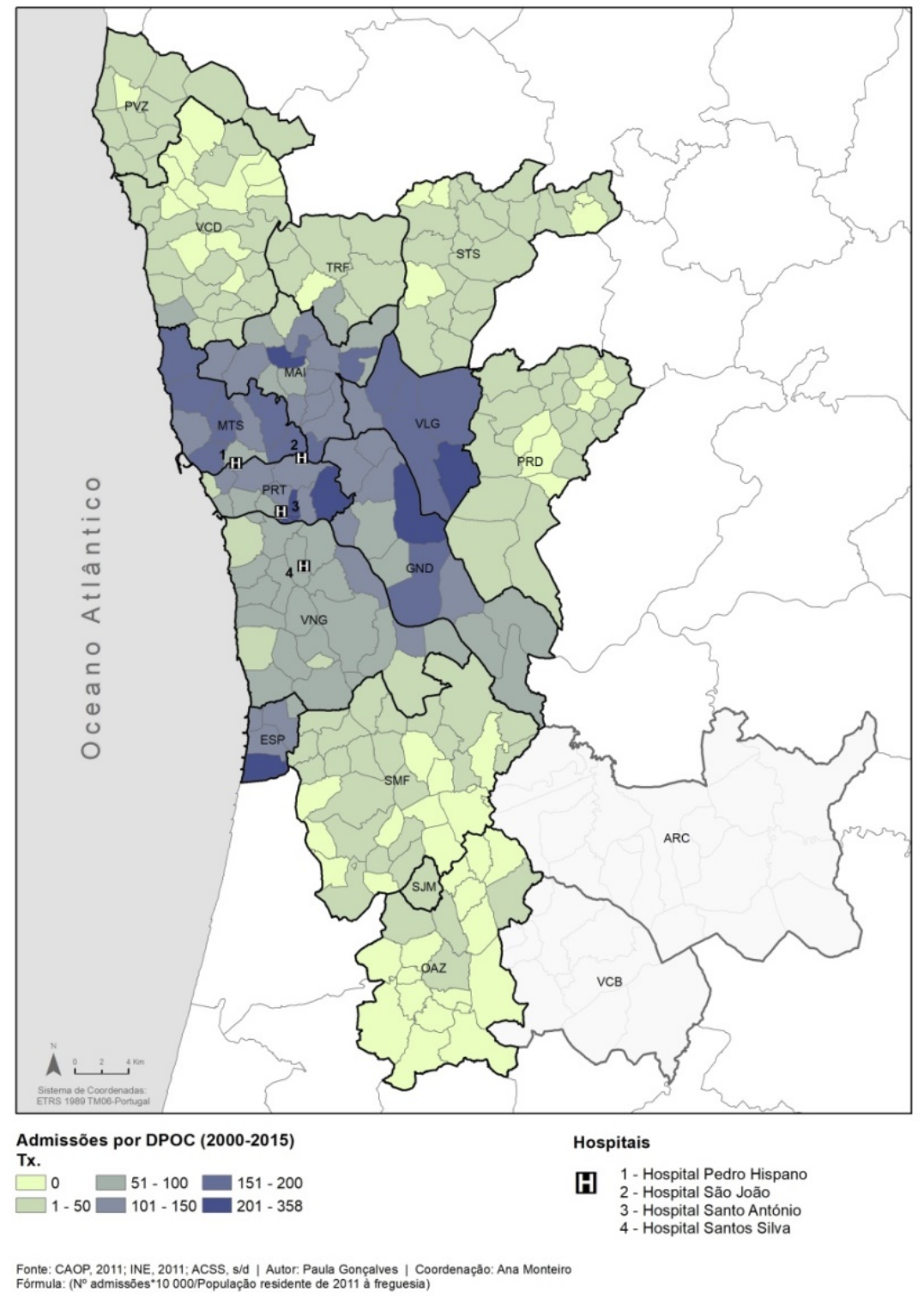

Figura 2: Taxa de admissão por DPOC nas freguesias da AMP entre 2000 e 2015.

Dentro das 14.079 admissões por DPOC entre 2000 e 2015 na AMP, 95 ocorreram entre os jovens, 3.767 entre os adultos e 10.217 entre os idosos. Estes números já revelam como a prevalência da enfermidade é maior no grupo com idade mais avançada, porém, ao relativizar os valores com base nos grupos etários do Censo de 2011, verifica-se como o número de admissões por DPOC entre os idosos é, aproximadamente, 12 vezes maior do que no grupo dos adultos e, quase, 100 vezes maior do que entre os jovens. A idade é um fator de risco associado à DPOC, fato comprovado por diversos estudos, que demonstram que a doença é mais recorrente no grupo dos idosos (e.g. Campos e Lemos, 2009, p. 301; Wang et al., 2010; Wichmann et al., 2011; Viveiros, 2014). Neste sentido, de modo geral, a Figura 3c exibe como nas freguesias do Porto e de Espinho, as maiores taxas de admissão mostradas na Figura 2, coincidem com a população mais envelhecida destas áreas. 

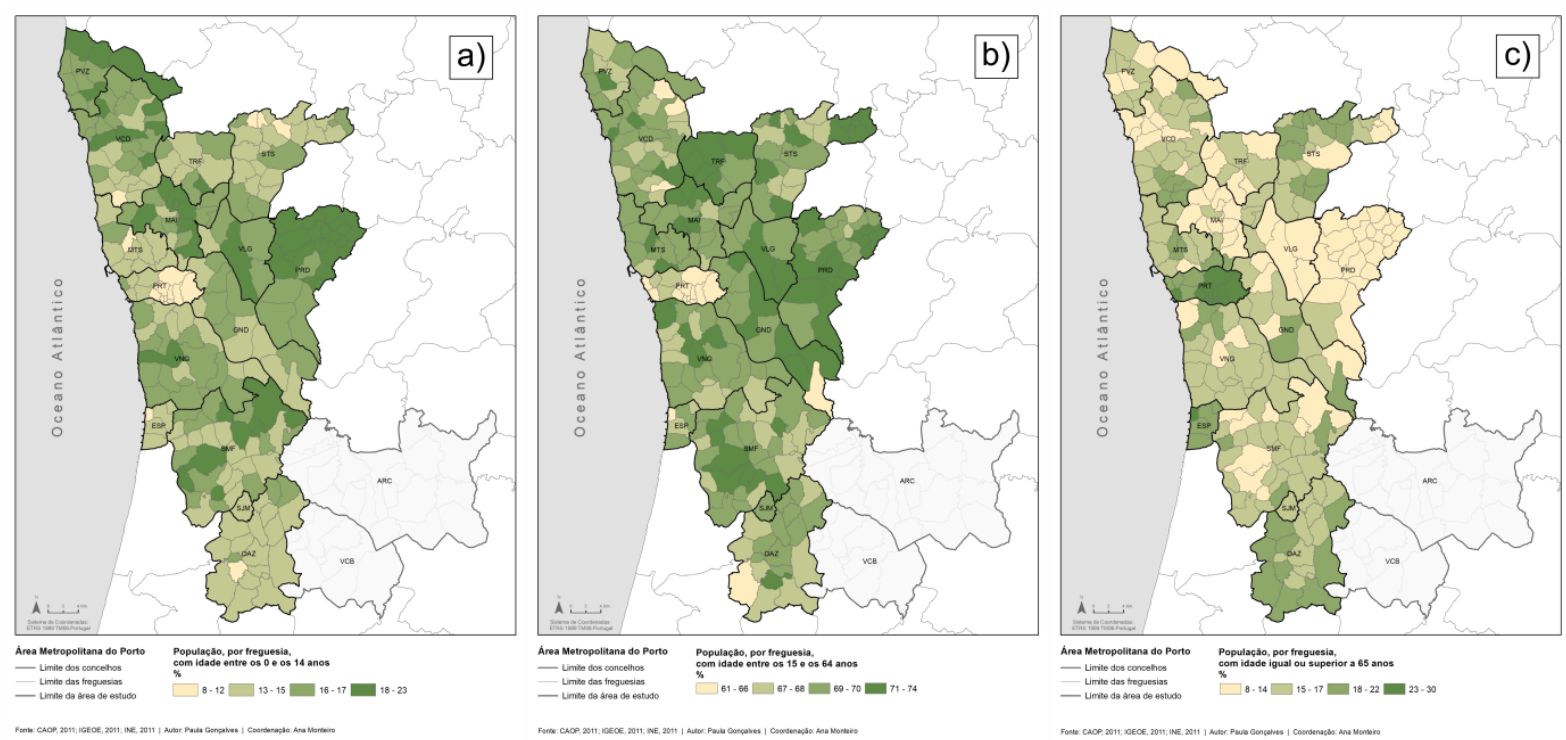

Figura 3: População por grupo etário (\%) distribuída nas freguesias da AMP. a) Jovens (0 a 14 anos). b) Adultos ( 15 a 64 anos). c) Idosos ( $\geq 65$ anos).

Fonte: INE, 2011.

Em Maia, Matosinhos e Gondomar, algumas freguesias também demonstram esta associação. $\mathrm{O}$ caso em que a relação entre idosos e DPOC não se apresenta tão claro é no concelho de Valongo e suas respectivas freguesias (Figura 3c).

No que concerne ao sexo das admissões por DPOC na AMP, no decurso dos anos avaliados, $9.057(64,3 \%)$ são do sexo masculino e $5.022(35,7 \%)$ do sexo feminino. A AMP no último Censo (INE, 2011), sem os concelhos de Arouca e Vale de Cambra, tinha uma população de $817.038(47,7 \%)$ homens e $897.263(53,3 \%)$ mulheres. Isto reforça ainda mais a predominância da patologia sob os homens, uma vez que os mesmos são minoria na área. A prevalência das admissões por DPOC no sexo masculino concorda com o verificado por Baldaia (1999) e por Bárbara et al. (2013), por exemplo. Bárbara et al. (2013), para Lisboa, atribuem a maior preponderância da DPOC entre os homens pelo fato dos mesmos serem ou terem sido maiores consumidores de tabaco.

Praticamente todo o concelho de Espinho apresenta-se como um sítio problemático, enfatizando a associação entre a DPOC, os idosos e os aspectos socioeconómicos precários, pois, a taxa de analfabetismo em toda sua área varia de 6,5 a $8,3 \%$, sendo esta a taxa mais crítica e; a taxa de desemprego oscila entre 15,4 a $27,1 \%$, ou seja, nas duas piores classes possíveis com base nos dados do Censo de 2011 (INE, 2011). Outro concelho que demonstra a mesma conjuntura é Gondomar, especificamente na freguesia que possui a maior taxa de admissão por DPOC, pois a mesma também está dentro da pior classe de taxa de desemprego e com uma alta taxa de analfabetismo $(4,5-6,4 \%)$. De modo geral, as freguesias localizadas na porção oriental do Porto, área que denota altas taxas de admissões pela patologia 
investigada, são as mesmas áreas que apresentam os maiores índices de desemprego e considerável taxa de analfabetos, assim como mais idosos (Figura 4).
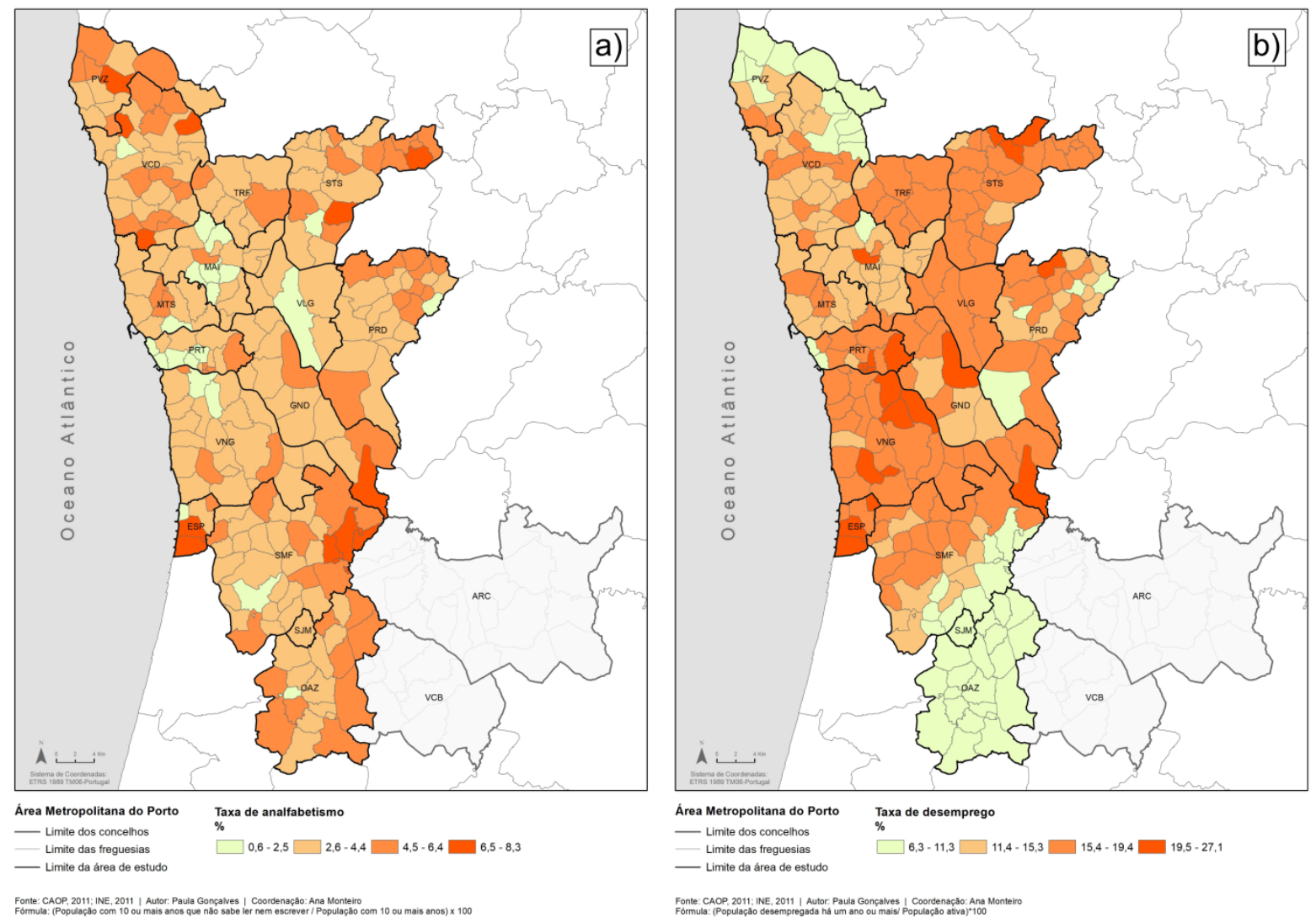

Figura 4: Aspectos socioeconómicos investigados na AMP. a) Taxa de analfabetismo (\%). b) Taxa de desemprego (\%).

Fonte: INE, 2011.

As freguesias centrais de Matosinhos possuem taxas socioeconómicas, com base nos aspectos aqui investigados, débeis. $\mathrm{O}$ analfabetismo e o desemprego, nestes sítios, estão dentro da segunda pior classe, variando de 4,5 - 6,4\% e 15,4 - 19,4\%, respectivamente. De modo similar ao que ocorre em Matosinhos, a freguesia central do concelho de Maia está dentro da segunda pior taxa de analfabetismo e da pior taxa de desempregados. Valongo, no que diz respeito ao analfabetismo, não está dentro das piores classes, porém, o concelho inteiro expõe a segunda pior taxa de desemprego (15,4 - 19,4\%), o que revela um problema social (Figura 4). Tais áreas citadas nestes três concelhos são aquelas em que a taxa de admissão por DPOC se apresenta mais adversa para a saúde da população (Figura 2). Em Vila Nova de Gaia, concelho em que a taxa de admissão por DPOC não é tão problemática como nos concelhos vizinhos, há uma freguesia a leste que denota um impacto mais elevado (101 - 150) (Figura 2), coincidindo com a pior taxa de desemprego cartografada (Figura 4b). Além destas mencionadas, há outras freguesias dentro dos demais 
concelhos da AMP em que os dados socioeconómicos averiguados são precários, mas, não estão associadas às taxas de admissão mais elevadas por DPOC.

Baldaia (1999), ao estudar 76 admissões por DPOC no Hospital São João na AMP, entre novembro de 1996 e novembro de 1997, verificou que os indivíduos mais afetados eram provenientes de condições socioeconómicas desfavorecidas e, na grande maioria, com idade entre 61 e 70 anos. Outros autores também salientam que o status socieconómico é um importante determinante das condições de saúde, incluindo os problemas por conta da DPOC (e.g. Gershon et al., 2012; Grigsby et al., 2016; Kocabas, Ozyilmaz, 2016). Gershon et al. (2012), por meio de revisão sistemática, concluíram que a associação entre DPOC e condições socieconómicas são inversas na grande maioria dos casos. Isto é, os indivíduos dos estratos socieconómicos mais baixos são os mais afetados pela enfermidade. Os autores ainda expõem que as pessoas nestas condições possuem, pelo menos, duas vezes mais chances de serem impactadas pela doença. Grigsby et al. (2016), ao investigarem 11.042 casos de DPOC na Argentina, Bangladesh, Chile, Peru e Uruguai, observaram menores taxas da doença nas pessoas com maior escolaridade e também com maior renda familiar. Resumidamente, a partir de um índice composto, os autores afirmam que a possibilidade de ter DPOC é maior em pessoas com piores índices socioeconómicos.

Embora Portugal tenha melhores índices socioeconómicos do que os países analisados por Gershon et al. (2016), é sabido que problemas deste cunho existem na maior parte dos países. Deste modo, há como fazer uma analogia com os aspectos socioeconómicos investigados aqui (Figura 4), pois se supõe que áreas com mais analfabetos e mais desempregados possuam maior vulnerabilidade social e, consequentemente, maiores chances que suas populações adoeçam.

As análises por freguesia foram feitas para espacializar a DPOC na AMP, uma vez que a base de dados das admissões por patologia não apresenta dados socioeconómicos dos indivíduos, impossibilitando uma possível inclusão de tais aspectos em análises estatísticas. Além disso, não foi viável ter acesso aos dados de tabagismo e de rendimentos por freguesia, sendo assim, as análises baseadas nestes importantes fatores de risco associados à DPOC, conforme bibliografias referenciadas, não puderam ser realizadas.

\subsection{Análises estatísticas entre as OdC, as OdF e a DPOC}

Para o Porto e concelhos vizinhos, a partir da estação climatológica da Serra do Pilar, de 2000 a 2015, Alves et al. (no prelo) detectaram 101 OdC e 27 OdF. Porém, com as sobreposições verificadas aqui por conta do gap de 14 dias, as OdC foram reduzidas a 71 eventos, ao passo que as OdF diminuíram para 23 casos. A distribuição temporal das 14.079 admissões por DPOC entre 2000 e 2015 na AMP pode ser visualizada na Figura 5. Vale ressaltar que a análise de RLM apenas incluiu os casos de admissões que ocorreram até 14 dias posteriores ao último dia da onda e não todas as apresentadas no gráfico abaixo (Figura 5). 


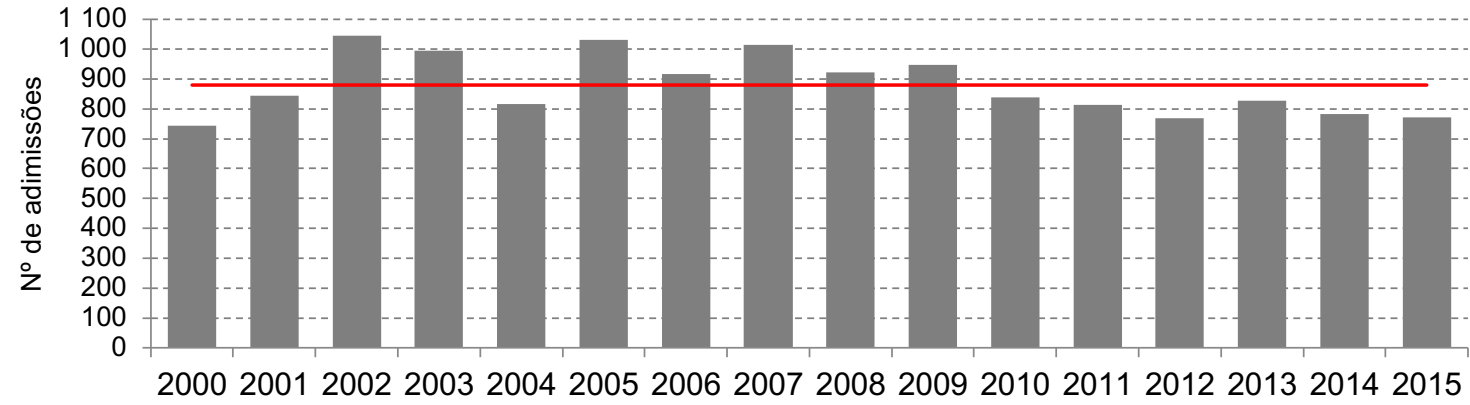

Figura 5: Número de admissões por DPOC na AMP entre 2000 e 2015. Totais anuais e a média da série (-).

Fonte: ACSS, s/d.

A média anual de admissões por DPOC na AMP ao longo dos anos estudados foi de $\sim 880$ casos. A primeira metade da série apresenta, ligeiramente, mais registros do que a segunda. Contudo, a distribuição é relativamente homogénea em todo o período (Figura 5). Dos 14.079 casos, a maioria ocorreu no inverno, com 5.129 (36,2\%); na sequência na estação primaveril com $3.698(26,2 \%)$; depois no outono com 2.907 $(20,6 \%)$ admissões e; por último, na estação do verão com 2.345 (17\%). Do montante geral das admissões investigadas, $35,2 \%$ ocorreram dentro do intervalo estabelecido para as ondas ( 2.057 dias). Estes números da AMP evidenciam como a DPOC é uma enfermidade ligada ao inverno e/ou, consequentemente, aos períodos mais frios na área. Tal característica ligada à sazonalidade das doenças respiratórias já é apontada por diversos autores, que indicam que as infecções respiratórias tendem a distribuir-se desta forma, sendo mais comuns durante o inverno nos climas temperados, como é o caso do norte de Portugal (Soebiyanto et al., 2010; Fisman, 2012; Van Noort et al., 2012; Monteiro et al., 2013). Entretanto, eventos de calor extremo também podem impactar negativamente tais enfermidades (Monteiro et al., 2013b; Hansel et al., 2015).

A Figura 6a demonstra os valores médios, máximos e mínimos da taxa de internação por DPOC ocorridas dentro do período de influência das OdC e das OdF detectadas. Observa-se que o maior pico na taxa de admissão referente às OdC foi de 14,2 casos, este valor foi registrado em um período de sobreposição de três ondas no inverno de 2008. Mesmo com a maior taxa de admissão acontecendo nas OdC, verifica-se que, em média, as OdF $(3,18)$ possuem mais casos de DPOC do que as OdC $(3,07)$. Mesmo com a média da duração das $\operatorname{OdC}(\sim 6,7)$ sendo, consideravelmente, maior do que a das $\operatorname{OdF}(\sim 4,1)$ (Figura $6 \mathrm{~b})$, a condição de duração do evento extremo de calor não explica ou reflete tão claramente os impactos na DPOC (Figura 6a); mostrando como o frio, mesmo sendo menos duradouro, impacta mais na patologia. $\mathrm{O}$ extremo máximo de duração na OdC ocorreu com a sobreposição de quatro eventos, na primavera de 2011, com 27 dias (Figura 6b). 

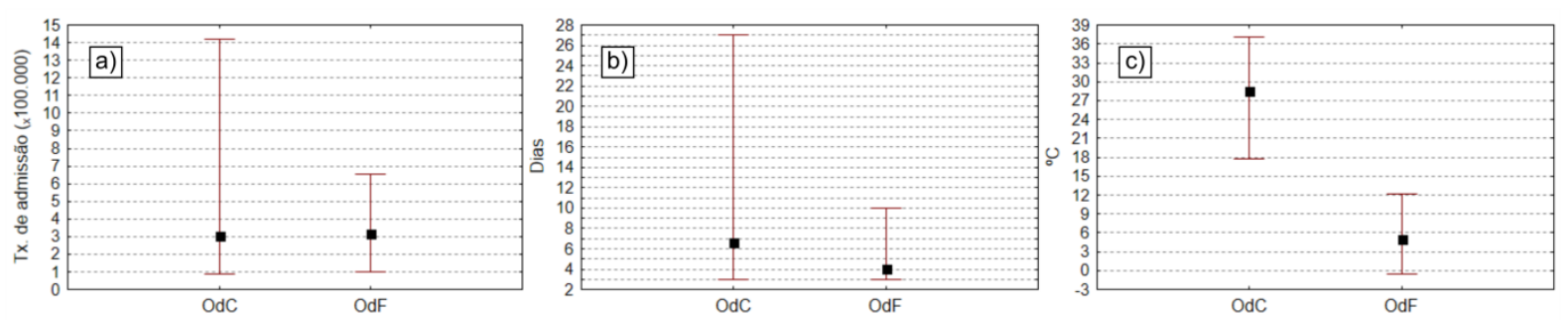

Figura 6: a) Box-Whiskers da taxa de admissão nas OdC e nas OdF. b) Box-Whiskers da duração das OdC e das OdF. c) Box-Whiskers da intensidade das OdC e das OdF. Os valores médios são representados por (·) e o Mín-Máx por (I).

Com relação à intensidade dos eventos (Figura 6c), nota-se que a média da temperatura do ar nas $\mathrm{OdC}$ é de $28,5^{\circ} \mathrm{C}$, ao passo que o valor máximo médio é de $37,1^{\circ} \mathrm{C}$ e foi verificado numa onda ocorrida no verão de 2003. Esta OdC é tratada como um evento extraordinário em Portugal (e.g. Marto, 2005; Montero, 2010; Cunha, Leal, 2013), coincidindo com o analisado aqui. Por sua vez, as OdF têm uma média de intensidade no valor de $5^{\circ} \mathrm{C}$, evidenciando as condições consideráveis de frio na AMP, lembrando que a duração mínima para detectar uma onda é de três dias consecutivos. Além da média, o extremo mínimo médio registrado nas OdF da série é de $-0,6^{\circ} \mathrm{C}$, ocorrido no inverno de 2012. Este episódio de frio extremo é estudado e documentado por Luo et al. (2014). É possível observar ainda que, nos valores médios para a intensidade, há um intervalo de temperatura "confortável" termicamente entre, aproximadamente, $12^{\circ} \mathrm{C}$ e $18^{\circ} \mathrm{C}$ (Figura 6c), pois não há registro de admissões por DPOC dentro da influência dos eventos de OdC e OdF nestas intensidades.

Dos três parâmetros existentes para as $\mathrm{OdC}$, apenas a $S m$ não fez parte da análise de RLM, por não ter apresentado correlação estatisticamente significativa ao nível de $\alpha=5 \%$ (Tabela II). Sendo assim, pelo modelo de RLM proposto para a OdC, foi possível identificar que, tanto a duração quanto a intensidade, compõem o modelo, explicando $68 \%\left(r^{2}\right)$ da variável dependente $(y)$. Ou seja, quase $70 \%$ da taxa diária de admissões por DPOC na AMP ocorridas durante a OdC são explicadas por ambas as variáveis em conjunto. Porém, a maior contribuição (peso) no modelo fica por conta do parâmetro duração (Tabela III).

Tabela III: Sumário da RLM para a variável dependente: Tx. de admissão por DPOC - OdC na AMP entre 2000 e 2015

\begin{tabular}{lrrrc}
\hline \multicolumn{1}{c}{$n=71$} & \multicolumn{1}{c}{$b$} & Erro Padrão de $b$ & \multicolumn{1}{c}{$t$} & $\mathrm{p}$-valor \\
\hline Intercepto & 6,346631 & 0,754706 & 8,40941 & $<0,0001^{*}$ \\
Duração & 0,242556 & 0,024122 & 10,05548 & $<0,0001^{*}$ \\
Intensidade & $-0,171746$ & 0,025422 & $-6,75576$ & $<0,0001^{*}$
\end{tabular}

Valores do teste: $r^{2}=0,68(68 \%) ; r^{2}$ ajustado = 0,66 (66\%); p-valor<0,0001; Erro Padrão estimado $=$ 1,182; Contribuição: Dur.>Int.. ( $\left.{ }^{*}\right)$ significativo ao nível de $\alpha=5 \%$.

A Figura 7 mostra os resíduos padrão da taxa de admissão durante os eventos de OdC, observa-se que apenas três valores estão fora do intervalo -2 e 2 , todavia, estes 
não são outliers por erros registrados, mas sim taxas resultantes dos eventos em que ocorreu sobreposição de ondas. Portanto, optou-se por não excluí-los, uma vez que são dados reais e podem repetir-se.

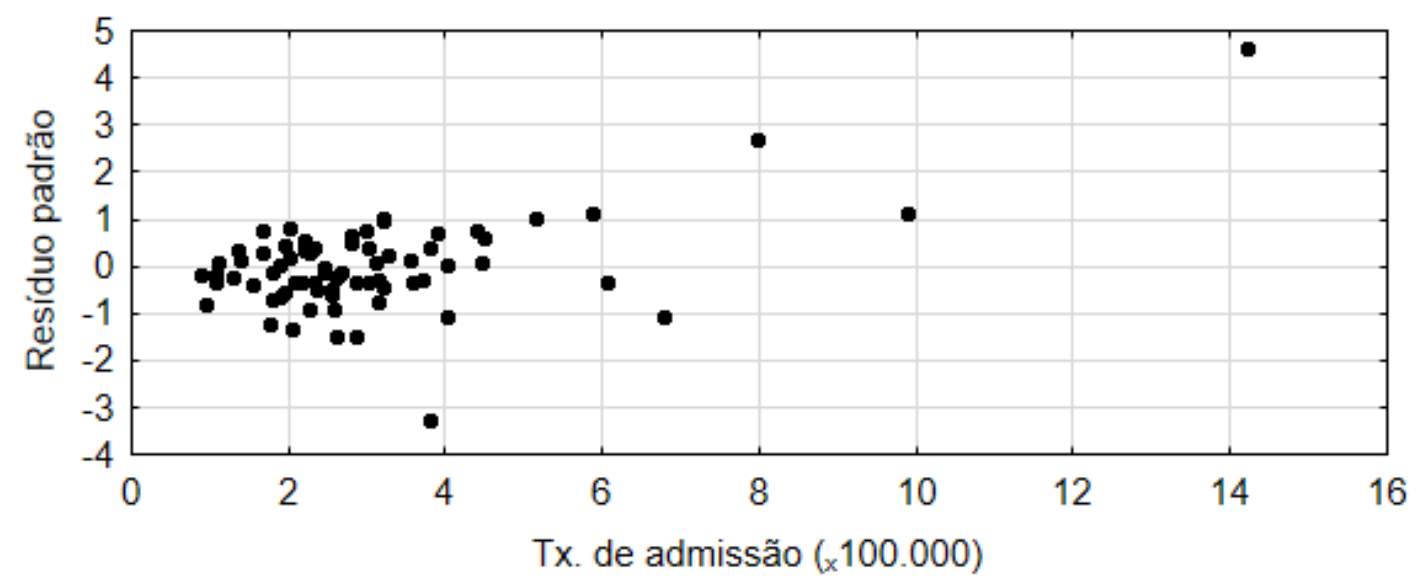

Figura 7: Resíduos padrão da taxa de admissão por DPOC durante os eventos de OdC - AMP entre 2000 e 2015.

Ao olhar os resultados do modelo de RLM acima, há como afirmar que os maiores impactos sob influência da OdC na enfermidade ocorrem nas situações em que os eventos são mais duradouros, ou seja, o parâmetro duração é o que mais contribui na explicação na taxa de admissão por DPOC. O fato de serem eventos de OdC, ou seja, de extremos de temperaturas elevadas, já é suficiente para desencadear problemas de saúde por si só e também, possivelmente, por apresentar ligações com os poluentes (Meng et al., 2012). Outras pesquisas ao analisarem os impactos das OdC nos casos de DPOC também evidenciam a associação entre as altas temperaturas e o incremento da morbimortalidade, sem relações a partir dos poluentes atmosféricos (Monteiro et al., 2013b; Åström et al., 2015; Hansel et al., 2015). D'Ippoliti et al. (2010) e Åström et al. (2013) revelam que os extremos de temperatura persistentes e anómalos sazonalmente, durante uma OdC, podem causar um acréscimo notável nas doenças respiratórias, com maior evidência nestes períodos. O impacto destas situações é conhecido como "efeito de adição da onda de calor" (Wichmann et al., 2011), gerando problemas de pressão sanguínea e respiratórios, incluindo a DPOC.

Além dos impactos diretos que a duração e a intensidade das OdC geram na saúde humana através da DPOC, por exemplo, as temperaturas elevadas também são responsáveis pelo aumento no nível de vários poluentes atmosféricos, especialmente em zonas com baixa qualidade do ar (Meng et al., 2012). Pesquisas feitas em distintos países europeus revelaram que os efeitos da poluição do ar são mais impactantes nos períodos de extremo de calor em comparação com os de frio (Dessai, 2003; Laaidi et al., 2006; Meng et al., 2012). A diminuição da qualidade do ar pode gerar um aumento nos casos de asma e DPOC (Sarfati e Abouzaid, 2009). Isto contribui para justificar 
os resultados encontrados aqui, mas, o efeito do calor extremo isoladamente já é o suficiente para impactar negativamente a DPOC.

Hansel et al. (2015) lembram que muitas pesquisas evidenciam os impactos que a exposição aos extremos de calor apresenta à saúde das pessoas, especialmente aos idosos e as pessoas com doenças respiratórias, incluindo a DPOC. Braga et al. (2002) ao estudarem 12 cidades estadunidenses, estimaram que os efeitos das temperaturas elevadas durante o verão podem aumentar o risco de morte atribuível à DPOC em até $25 \%$. Lin et al. (2009), para Nova lorque, concluíram que o risco de hospitalização por DPOC aumenta $7,6 \%$ a cada incremento de $1^{\circ} \mathrm{C}$ acima de um limiar observado para a temperatura. Anderson et al. (2013) investigando 12,5 milhões de idosos em 213 localidades urbanas estadunidenses, verificaram um aumento de $4,7 \%$ no risco de hospitalização por DPOC a cada aumento de $\sim 5,5^{\circ} \mathrm{C}$ na temperatura ambiente. Hansel et al. (2015), do ponto de vista fisiológico, indicam que os mecanismos pelos quais a exposição ao calor afeta negativamente a DPOC não são totalmente compreendidos, todavia, além das respostas termorregulatórias, há um efeito direto do calor no sistema respiratório. Estudos em asma sugerem que a respiração com o ar quente e húmido pode resultar em broncoconstrição (Hayes et al., 2012). Ou seja, as OdC apenas por serem eventos com altas temperaturas em diferentes estações do ano prejudicam a saúde humana, neste caso, refletida na DPOC. Isto explica o motivo pelo qual as OdC exibiram associações significativas e consideravelmente explicativas nas taxas de admissão por DPOC na AMP entre 2000 e 2015.

O gráfico de dispersão entre a duração e a taxa de admissão por DPOC (Figura 8a) indica que, de maneira geral, as situações que demonstram maiores taxas de admissão são aquelas em que as OdC são mais duradouras. E, conforme citado anteriormente, as OdC menos intensas dentro de um intervalo de $\sim 18^{\circ} \mathrm{C}$ e $\sim 36^{\circ} \mathrm{C}$ (Figura 6c), tendem a apresentar maiores taxas (Figura 8b). Este intervalo apresenta uma oscilação considerável, pois os eventos foram analisados anualmente, uma vez que não ocorrem OdC (OdF) somente nos períodos quentes (frios).
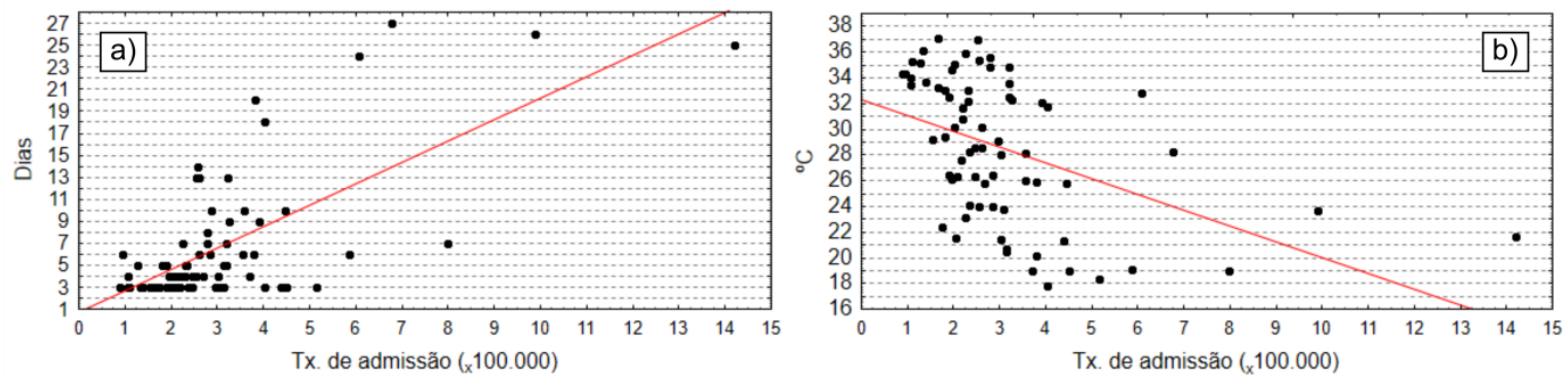

Figura 8: a) Gráfico de dispersão entre Duração das OdC e Tx. de admissão por DPOC. b) Gráfico de dispersão entre a Intensidade das OdC e Tx. de admissão por DPOC - AMP entre 2000 e 2015.

Já para a análise de RLM, todos os parâmetros das OdF foram avaliados no modelo, uma vez que os testes de correlação feitos anteriormente exibiram significância 
estatística $(\alpha=5 \%)$ com a patologia (Tabela II). Porém, ao realizar o teste, verificouse que a variável severidade média $(\mathrm{Sm})$ não contribuiu significativamente na prevalência da DPOC, visto que o p-valor foi maior do que 0,05. Com base nisto, a variável dependente $(y)$ é explicada em $82 \%\left(r^{2}\right)$ pelos parâmetros intensidade e duração das OdF (Tabela IV). Deste modo, é possível afirmar que as OdF explicam $82 \%$ da prevalência da DPOC na AMP entre 2000 e 2015, a partir da sinergia entre a intensidade e a duração. Ressalta-se que, neste caso, a intensidade contribuiu mais do que a duração.

Tabela IV: Sumário da RLM para a variável dependente: Tx. de admissão por DPOC - OdF na AMP entre 2000 e 2015

\begin{tabular}{lrrrr}
\hline \multicolumn{1}{c}{$n=23$} & \multicolumn{1}{c}{$b$} & Erro Padrão de $b$ & \multicolumn{1}{c}{$t$} & \multicolumn{1}{c}{$\mathrm{p}$-valor } \\
\hline Intercepto & 2,709860 & 0,692155 & 3,91510 & $0,000930^{*}$ \\
Intensidade & $-0,239321$ & 0,037713 & $-6,34590$ & $0,000004^{*}$ \\
Duração & 0,387416 & 0,095372 & 4,06215 & $0,000665^{*}$ \\
Severidade & 0,091487 & 0,378245 & 0,24187 & 0,811471 \\
\hline
\end{tabular}

Valores do teste: $r^{2}=0,82(82 \%) ; r^{2}$ ajustado = 0,79 (79\%); $p$-valor<0,0001; Erro Padrão estimado = 0,760 ; Contribuição: Int. $>$ Dur.. $\left({ }^{*}\right)$ significativo ao nível de $\alpha=5 \%$.

Ao observar os resíduos padrão da taxa de admissão durante os eventos de OdF (Figura 9), constata-se que somente um valor está fora do intervalo -2 e 2. Mas, conforme citado anteriormente para as $\mathrm{OdC}$, tais valores são taxas reais resultantes dos eventos em que também ocorreu sobreposição de ondas. Deste modo, os registros não foram excluídos.

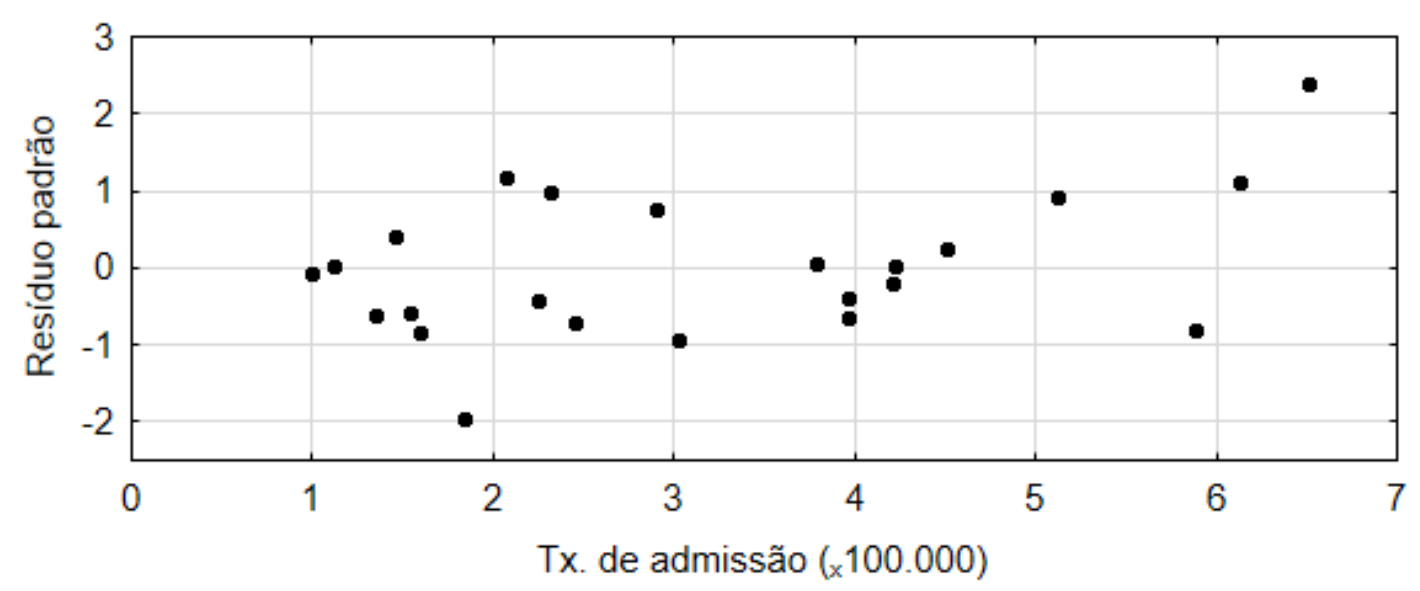

Figura 9: Resíduos padrão da taxa de admissão por DPOC durante os eventos de OdF - AMP entre 2000 e 2015.

Embora trate da mortalidade, uma pesquisa realizada em 15 cidades da Europa, avaliando o efeito da diminuição da temperatura em curto prazo, demonstrou que a diminuição de $1^{\circ} \mathrm{C}$ na temperatura do ar está associada a 3,30\% no aumento da 
mortalidade por doenças respiratórias. Portanto, a intensidade, mesmo que com pouca duração, pode surtir efeito direto na saúde humana (Analitis et al., 2008). Jenkins et al. (2012), ao estudarem a sazonalidade e os possíveis determinantes nos casos de exacerbações por DPOC em diversos países do mundo, nos dois hemisférios, verificaram que aqueles localizados nos ambientes extratrópicos possuem relação clara com os invernos, ou seja, com as baixas temperaturas, independentemente de outros fatores de risco. Tal informação enfatiza a importância da intensidade das temperaturas na enfermidade investigada. Segundo os autores, nenhum padrão sazonal aconteceu nos trópicos. Hansel et al. (2015), com base na fisiologia humana, indicam que os casos de associação entre frio e DPOC estão ligados à redução da função pulmonar. Casos de broncoconstrição e inflamação podem ocorrer em situações de exposição ao frio (Koskela et al., 1996; Donaldson et al., 1999), mas, além destes, pesquisas mais recentes sugerem um papel importante para a hipersecreção mucosa como potencial mediador da resposta da DPOC aos casos de frio (Li et al., 2011).

A dispersão entre a duração das OdF e a taxa de admissão por DPOC exibe que, salvo alguns casos, o impacto na patologia não depende tanto da duração, diferentemente das OdC. Mas, ainda assim há como perceber a influência da duração na taxa de admissão, especialmente nos eventos mais duradouros, com períodos acima de quatro dias (Figura 10a). De modo semelhante às OdC, porém, com mais expressão, a intensidade nas OdF demonstram claramente o impacto na patologia (Figura 10b).
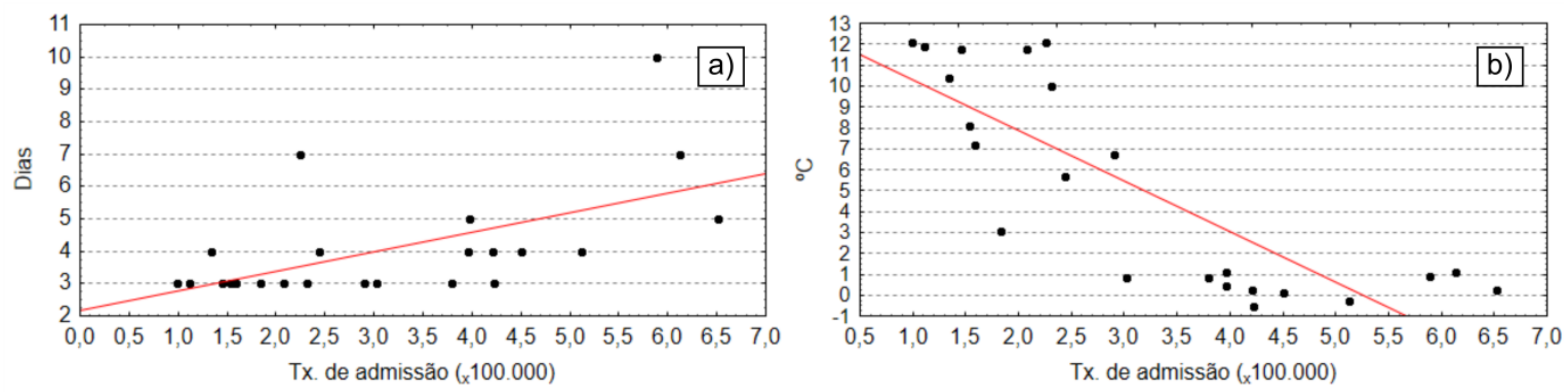

Figura 10: a) Gráfico de dispersão entre Duração das OdF e Tx. de admissão por DPOC. b) Gráfico de dispersão entre a Intensidade das OdF e Tx. de admissão por DPOC. - AMP entre 2000 e 2015.

Há como verificar ainda que, de modo geral, abaixo dos $5^{\circ} \mathrm{C}$ existe uma prevalência das maiores taxas de admissão, com mais de 3,5 por OdF. Kumi e Ayres (2007) expõem que a diminuição de $1^{\circ} \mathrm{C}$ nas temperaturas abaixo de $5^{\circ} \mathrm{C}$ está associada ao aumento de $10 \%$ das consultas médicas por DPOC. Além destes, Monteiro et al. (2013a), para os invernos entre 2000 e 2007 no Porto, apontam que os casos de extremos de frio $\leq 5^{\circ} \mathrm{C}$ podem ser mais significativos no aumento da morbidade por DPOC. 


\section{Conclusões}

A localização dos hospitais cujos dados foram analisados e a concentração destes na área central da AMP pode ter tido influência direta na distribuição espacial da taxa de admissão por DPOC, fazendo com que, de maneira geral, as freguesias nas cercanias obtivessem os maiores índices. Do ponto de vista da caracterização das admissões por DPOC na AMP e os aspectos da população avaliados, conclui-se que, a doença está associada às freguesias com taxas socioeconômicas mais precárias e, geralmente, com a população mais envelhecida, especialmente em Espinho e nas freguesias orientais do Porto. Em Gondomar também há uma freguesia que chama atenção pela associação entre alta taxa de admissão e baixos níveis sociais, todavia, sem ter uma população tão idosa. Estas constatações, na maior parte dos casos, estão em linha com a bibliografia sobre a temática.

Atestou-se que a DPOC na AMP é uma enfermidade que responde aos efeitos do clima, notadamente, as OdC e as OdF. Embora se tenha mais eventos de OdC nos anos analisados, ainda assim as OdF, na média, causam mais admissões. No que tange às OdF, a intensidade é mais importante do que a duração para a taxa de admissão por DPOC. Ou seja, eventos com menores temperaturas, mas, sem grandes durações causam mais impactos do que o contrário, especialmente em eventos com intensidade abaixo dos $5^{\circ} \mathrm{C}$. A associação entre DPOC e OdC também apresentou relação estatisticamente significativa, todavia, nos eventos de calor a duração é o parâmetro que mais contribui no modelo explicativo ajustado pela RLM. A intensidade em declínio na OdC também contribuiu para o aumento da taxa de admissão por DPOC, todavia, vale ressaltar que é o decréscimo dentro de um intervalo entre $\sim 36^{\circ} \mathrm{C}$ e $\sim 18^{\circ} \mathrm{C}$, ou seja, ainda sob incumbência de um extremo quente de acordo com o período do ano. Obviamente que uma OdC com intensidade de $\sim 18^{\circ} \mathrm{C}$ não foi registrada durante os períodos climatologicamente quentes para a AMP, porém, tal intensidade ocorreu durante os períodos frios e foi impactante, principalmente no inverno.

Os modelos elaborados com base nos parâmetros das OdC e das OdF, com exceção da severidade média, possibilitam a estimativa da taxa de admissão por DPOC na AMP a partir da intensidade e da duração, com confiança estatística atestada.

Verificou-se que há um período de "conforto" térmico no que tange às taxas de admissão por DPOC na AMP, pois não ocorreram registros entre $\sim 12^{\circ} \mathrm{C}$ e $\sim 18^{\circ} \mathrm{C}$. Contudo, não é que não existam admissões com tais intensidades, o fato é que não sucederam OdC e/ou OdF neste intervalo de temperatura no período investigado.

Por fim, ao verificar que a DPOC é uma doença impactante na AMP, com mais evidência em algumas áreas e que os efeitos da OdC e das OdF influenciam na enfermidade, sugere-se que estudos subsequentes venham a ser elaborados e que análises mais detalhadas com os aspectos socioeconómicos das localidades e/ou com as características dos enfermos sejam levadas a cabo. 


\section{Agradecimentos}

Este trabalho foi parcialmente financiado pelo FEDER "Factores de CompetitividadeCOMPETE", pela Fundação de Ciência e Tecnologia (FCT) através do projecto TRAPHIC (PTDC/ECM-URB/3329/2014, POCI-01-0145-FEDER-016729) e pela Comissão Europeia através do projeto H2020-LC-CLA-2019-2020_EXHAUSTION Exposure to heat and air pollution in Europe - cardiopulmonary impacts and benefits of mitigation and adaptation. $\mathrm{O} 2^{\circ}$ autor agradece ainda à Coordenação de Aperfeiçoamento de Pessoal de Nível Superior (CAPES) por sua bolsa de doutorado (Processo $n^{\circ}$ : 1696632). Os agradecimentos se estendem também à $\mathrm{Dr}^{a}{ }^{a}$ Fátima Cardoso (Administração Central dos Serviços de Saúde), ao Dr. Fernando José Oliveira Lopes (Hospital de São João), ao Dr. Alberto Freitas (CINTESIS) e ao Dr. Victor Prior (Instituto Português do Mar e da Atmosfera). Por último, os autores agradecem ao Acordo de Cooperação existente entre a UPorto (FLUP) e a UFSC (PPGG), o qual viabilizou o estágio dos doutorandos brasileiros no IGUP.

\section{Bibliografia}

ACSS - Administração Central do Sistema de Saúde. s/d. Dados. Serviço Nacional de Saúde. Consultado em: www.acss.min-saude.pt

Almeida, S. P., Casimiro, E., Calheiros, J. (2010). Effects of apparent temperature on daily mortality in Lisbon and Oporto, Portugal. Environ Health, 9:12. https://doi.org/10.1186/1476-069X-9-12

Alves, M. P. A., Silveira, R. B., Bitencourt, D. P., Franke, A. E. (2017). Onda de frio? Análise de diferentes métodos de identificação. Revista Brasileira de Climatologia, 21, 295-312. http://dx.doi.org/10.5380/abclima.v21i0.54821

Alves, M. P. A., Silveira, R. B., Monteiro, A., Bitencourt, D. P., Sousa, C. M. (no prelo). Reconstrução de dados e detecção de ondas de calor e de frio no Porto e concelhos vizinhos - Portugal. Territorium, 27(2), 20.

Analitis, A., Katsouyanni, K., Biggeri, A., Baccini, M., Forsberg, B., Bisanti, L., Kirchmayer, U., Ballester, F., Cadum, E., Goodman, P. G., Hojs, A., Sunyer, J., Tiittanen, P., Michelozzi, P. (2008). Effects of cold weather on mortality: results from 15 European cities within the PHEWE project. Am J Epidemiol, 168(12), 1397-408. https://doi.org/10.1093/aje/kwn266

Anderson, B. G., Bell, M. L. (2009). Weather-related mortality: how heat, cold, and heat waves affect mortality in the United States. Epidemiology, 20(2), 205-13. https://doi:10.1097/EDE.0b013e318190ee08

Anderson, G. B., Dominici, F., Wang, Y., McCormack, M. C., Bell, M. L., Peng, R. D. (20139. Heat-related emergency hospitalizations for respiratory diseases in the Medicare population. Am J Respir Crit Care Med, 187(10), 1098-1103. http://doi.org/10.1164/rccm.201211-19690C

Antunes, L., Silva, S. P., Marques, J., Nunes, B., Antunes, S. (2016). The effect of extreme cold temperatures on the risk of death in the two major Portuguese cities. Int J Biometeorol, 61(1), 127-35. http://doi.org/10.1007/s00484-016-1196-x 
Åström, C., Orru, H., Rocklöv, J., Strandberg, G., Ebi, K. L., Forsberg, B. (2013). Heat-related respiratory hospital admissions in Europe in a changing climate: a health impact assessment. BMJ Open, 3(1), e001842. http://dx.doi.org/10.1136/bmjopen-2012-001842

Åström, D. O., Schifano, P., Asta, F., Lallo, A., Michelozzi, P., Rocklöv, J., Forsberg, B. (2015). The effect of heat waves on mortality in susceptible groups: a cohort study of a mediterranean and a northern European City. Environmental Health, 14(1), 30. http://doi.org/10.1186/s12940-015-0012-0

Baldaia, J. (1999). Factores de ordem social, económica e cultural da DPOC em internamento hospitalar. Rev Portuguesa de Pneumologia, 5(1), 67-73. https://doi.org/10.1016/S08732159(15)30955-7

Ballester, F., Michelozzi, P., Iñiguez, C. (2003). Weather, climate, and public health. J Epidemiol Community Health, 57(10), 759-60. http://dx.doi.org/10.1136/jech.57.10.759

Bárbara, C., Rodrigues, F., Dias, H., Cardoso, J., Almeida, J., Matos, M. J., Simão, P., Santos, M., Ferreira, J. R., Gaspar, M., Gnatiu, L., Burney, P. (2013). Prevalência da doença pulmonar obstrutiva crónica em Lisboa, Portugal: estudo Burden of Obstructive Lung Disease. Rev Portuguesa de Pneumologia, 19(3), 96-105. https://doi.org/10.1016/j.rppneu.2012.11.004

Barnett, A. G., Hajat, S., Gasparrini, A., Rocklöv, J. (2012). Cold and heat waves in the United States. Environmental Research, 112, 218-24. https://doi.org/10.1016/j.rppneu.2012.11.004

Bitencourt, D. P., Fuentes, M. V., Franke, A. E., Silveira, R. B., Alves, M. P. A. (no prelo). The climatology of cold and heat waves in Brazil from 1961 to 2016. International Journal of Climatology, 29.

Braga, A. L., Zanobetti, A., Schwartz, J. (2002). The effect of weather on respiratory and cardiovascular deaths in 12 U.S. cities. Environ Health Perspect, 110(9), 859-863. http://doi.org/10.1289/ehp.02110859

Campos, H. S., Lemos, A. C. M. (2009). A asma e a DPOC na visão do pneumologista. Jornal Brasileiro de Pneumologia, 35, 301-309. http://dx.doi.org/10.1590/S1806$\underline{37132009000400003}$

COPD Foundation. s/d. What is COPD?. Consultado em: https://www.copdfoundation.org/What-is-COPD/Understanding-COPD/What-isCOPD.aspx

Cunha, L., Leal, C. (2013). Ondas de calor e ondas de frio no município de Torres Novas. Cadernos de Geografia Coimbra, 32, 81-93. Consultado em: https://impactumjournals.uc.pt/cadernosgeografia/article/view/2431

Daveau, S. (1995). Portugal Geográfico. Edições João Sá da Costa, Lisboa.

Dessai, S. (2003). Heat stress and mortality in Lisbon Part II. An assessment of the potential impacts of climate change. Int J Biometeorol, 48(1), 37-44. https://doi.org/10.1007/s00484003-0180-4

Diaz, J., Garcia, R., Lopez, C., Linares, C., Tobias, A., Prieto, L. (2005). Mortality impact of extreme winter temperatures. Int J Biometeorol, 49(3), 179-83. https://doi.org/10.1007/s00484-004-0224-4 
D'Ippoliti, D., Michelozzi, P., Marino, C., de'Donato, F., Menne, B., Katsouyanni, K., Kirchmayer, U., Analitis, A., Medina-Ramón, M., Paldy, A., Atkinson, R., Kovats, S., Bisanti, L., Schneider, A., Lefranc, A., Iñiguez, C., Perucci, C. A. (2010). The impact of heat waves on mortality in 9 European cities: results from the EuroHEAT project. Environ Health, 9(37), 9. https://doi.org/10.1186/1476-069X-9-37

Donaldson, G. C., Seemungal, T., Jeffries, D. J., Wedzicha, J. A. (1999). Effect of temperature on lung function and symptoms in chronic obstructive pulmonary disease. Eur Respir $\mathrm{J}$, 13(4), 844-849. http://doi.org/10.1034/j.1399-3003.1999.13d25.x

Durmaz, D., Goksu, E., Yildiz, G., Unal, A., Kartal, M., Oskay, A., Simsek, Y. (2015). The factors influencing relapse in patients presenting to the emergency department with COPD exacerbation. Turkish Journal of Emergency Medicine, 15(2), 59-63. https://doi.org/10.5505/1304.7361.2014.37791

Ferrari, U., Exner, T., Wanka, E. R., Bergemann, C., Meyer-Arnek, J., Hildenbrand, B., Amanda Tufman, A., Heumann, C., Huber, R. M., Bittner, M., Fischer, R. (2012). Influence of air pressure, humidity, solar radiation, temperature, and wind speed on ambulatory visits due to chronic obstructive pulmonary disease in Bavaria, Germany. Int J Biometeorol, 56(1), 137-43. https://doi.org/10.1007/s00484-011-0405-x

Fisman, D. (2012). Seasonality of viral infections: mechanisms and unknowns. Clin Microbiol Infect, 18(10), 946-54. https://doi.org/10.1111/j.1469-0691.2012.03968.x

Gasparrini, A., Guo, Y., Hashizume, M., Lavigne, E., Zanobetti, A., Schwartz, J., Tobias, A., Tong, S., Rocklöv, J., Forsberg, B., Leone, M., De Sario, M., Bell, M. L., Guo, Y. L., Wu, C., Kan, H., Yi, S., Coelho, M. S. Z. S., Saldiva, P. H. N. (2015). Mortality risk attributable to high and low ambient temperature: a multicountry observational study. The Lancet, 386(9991), 369-75. https://doi.org/10.1016/S0140-6736(14)62114-0

Geirinhas, J. L., Trigo, R. M., Libonati, R., Coelho, C. A., Palmeira, A. C. (2018). Climatic and synoptic characterization of heat waves in Brazil. Int J Climatol, 38(4), 1760-1776. https://doi.org/10.1002/joc.5294

Gershon, A. S., Dolmage, T. E., Stephenson, A., Jackson, B. (2012). Chronic obstructive pulmonary disease and socioeconomic status: a systematic review. Joumal COPD, 9(3), 216-26. https://doi.org/10.3109/15412555.2011.648030

Grigsby, M., Siddharthan, T., Chowdhury, M. A., Siddiquee, A., Rubinstein, A., Sobrino, E., Miranda, J. J., Bernabe-Ortiz, A., Alam, D., Checkley, W. (2016). Socioeconomic status and COPD among low- and middle-income countries. Int J Chron Obstruct Pulmon Dis, 11, 2497-507. https://doi.org/10.2147/COPD.S111145

Hammer, Ø., Harper, D. A. T., Ryan, P. D. (2001). PAST: Paleontological Statistics software package for education and data analysis. Palaeontologia Electronica, 4(1), 9. Consultado em: https://palaeo-electronica.org/2001 1/past/issue1 01.htm

Hansel, N. N., McCormack, M. C., Kim, V. (2015). The effects of air pollution and temperature on COPD. Journal COPD, 13(3), 372-379. https://doi.org/10.3109/15412555.2015.1089846

Hayes, D., Collins, P. B., Khosravi, M., Lin, R. L., Lee, L. Y. (2012). Bronchoconstriction triggered by breathing hot humid air in patients with asthma: role of cholinergic reflex. $\mathrm{Am}$ J Respir Crit Care Med, 185(11), 1190-1196. https://doi.org/10.1164/rccm.201201-00880C 
Huang, C., Barnett, A. (2014). Winter weather and health. Nature Climate Change, 4, 173. https://doi.org/10.1136/jech.57.10.784

Huynen, M. M., Martens, P., Schram, D., Weijenberg, M. P., Kunst, A. E. (2001). The impact of heat waves and cold spells on mortality rates in the Dutch population. Environ Health Perspect, 109(5), 463-70. https://doi.org/10.1289/ehp.01109463

Inagaki, R. K., Yamaguchi, M. H., Kassada, D., Matsuda, L. M., Marcon, S. S. (2008). A vivência de uma idosa cuidadora de um idoso doente crônico. Ciência, Cuidado e Saúde, 7, 4. http://dx.doi.org/10.4025/cienccuidsaude.v7i0.20802

INE - Instituto Nacional de Estatística (2011). Censos: Censo 2011. Consultado em: https://censos.ine.pt/xportal/xmain?xpgid=censos2011 apresentacao\&xpid=CENSOS

Ingole, V., Kovats, S., Schumann, B., Hajat, S., Rocklöv, J., Juvekar, S., Armstrong, B. (2017). Socioenvironmental factors associated with heat and cold-related mortality in Vadu HDSS: western India: a population-based case-crossover study. Int J Biometeorol, 61(10), 1797804. https://doi.org/10.1007/s00484-017-1363-8

Jenkins, C. R., Celli, B., Anderson, J. A., Ferguson, G. T., Jones, P. W., Vestbo, J., Yates, J. C., Calverley, P. M. A. (2012). Seasonality and determinants of moderate and severe COPD exacerbations in the TORCH study. Eur Respir J, 39(1), 38-45. https://doi.org/10.1183/09031936.00194610

Kimble, G. H. T. (1938). Geography in the Middle Ages. Methuen \& Company, Limited.

Kocabas, A., Ozyilmaz, E. (2016). The effects of socioeconomic status on health outcomes in patients with COPD. European Respiratory Journal, 48 (suppl 60), PA1030. https://doi.org/10.1183/13993003.congress-2016.PA1030

Koskela, H. O., Koskela, A. K., Tukiaineu, H. O. (1996). Bronchoconstriction due to cold weather in COPD. The roles of direct airway e ects and cutaneous reflex mechanisms. Chest, 110(3), 632-636. http://doi.org/10.1378/chest.110.3.632

Laaidi, M., Laaidi, K., Besancenot, J. P. (2006). Temperature-related mortality in France, a comparison between regions with different climates from the perspective of global warming. Int J Biometeorol, 51(2), 145-53. https://doi.org/10.1007/s00484-006-0045-8

Lacaz, C. S., Junior, W. S., Baruzzi, R. G. (1972). Introdução à geografia médica do Brasil. Editora Blücher.

Langford, I. H., Bentham, G. (1995). The potential effects of climate change on winter mortality in England and Wales. Int J Biometeorol, 38(3), 141-7. https://doi.org/10.1007/BF01208491

Li, M., Li, Q., Yang, G., Kolosov, V. P., Perelman, J. M., Zhou, X. D. (2011). Cold temperature induces mucin hypersecretion from normal human bronchial epithelial cells in vitro through a transient receptor potential melastatin 8 (TRPM8)-mediated mechanism. J Allergy Clin Immunol, 128(3), 626-634.e5. http://doi.org/10.1016/j.jaci.2011.04.032

Lin, G., Zacharek, M. (2012). Climate change and its impact on allergic rhinitis and other allergic respiratory diseases. Current Opinion Otolaryngology Head Neck Surg, 20(3), 188193. https://doi.org/10.1097/MOO.0b013e3283524b1

Lin, S., Luo, M., Walker, R. J., Liu, X., Hwang, S. A., Chinery, R. (2009). Extreme high temperatures and hospital admissions for respiratory and cardiovascular diseases. Epidemiology, 20(5), 738-746. http://doi.org/10.1097/EDE.0b013e3181ad5522 
Luo, D., Yao, Y., Feldstein, S. B. (2014). Regime transition of the North Atlantic Oscillation and the extreme cold event over Europe in January-February 2012. Monthly Weather Review, 142(12), 4735-4757. https://doi.org/10.1175/MWR-D-13-00234.1

Marto, N. (2005). Ondas de calor: impacto sobre a saúde. Acta Medica Portuguesa, 18, 467474. Consultado em: https://www.actamedicaportuguesa.com/revista/index.php/1063/731

Maunder, W. J. (1970). The value of the weather. London: Methuen \& Co Ltd.

Meng, X., Zhang, Y., Zhao, Z., Duan, X., Xu, X., Kan, H. (2012). Temperature modifies the acute effect of particulate air pollution on mortality in eight Chinese cities. Sci Total Environ, 435-436, 215-21. https://doi.org/10.1016/j.scitotenv.2012.07.008

Miller, A. A. (1953). Climatology. Methuen: London, University of Reading.

Ministério da Saúde - Portugal (2017a). Relatório anual: acesso a cuidados de saúde nos estabelecimentos do SNS e entidades convencionadas. Lisboa. Consultado em: https://www.sns.gov.pt/wpcontent/uploads/2018/06/Relatorio Acesso SNS 2017 v.final .pdf

Ministério da Saúde - Portugal (2017b). DPOC em números: Portugal e Europa. Lisboa. Consultado em: https://www.mgfamiliar.net/LiteratureRetrieve.aspx?ID=132081

Ministério da Saúde - Portugal (2018). Retrato da saúde, Portugal. Lisboa. Consultado em: https://www.sns.gov.pt/wp-content/uploads/2018/04/RETRATO-DA-

SAUDE 2018 compressed.pdf

Monteiro, A., Carvalho, V., Velho, S., Sousa, C. (2012). Assessing and monitoring urban resilience using COPD in Porto. Science of The Total Environment, 414, 113-119. https://doi.org/10.1016/j.scitotenv.2011.11.009

Monteiro, A., Carvalho, V., Gois, J., Sousa, C. (2013a). Use of "Cold Spell" indices to quantify excess chronic obstructive pulmonary disease (COPD) morbidity during winter (November to March 2000-2007): case study in Porto. Int $J$ Biometeorol, 57(6), 857-870. https://doi.org/10.1007/s00484-012-0613-z

Monteiro, A., Carvalho, V., Oliveira, T., Sousa, C. (2013b). Excess mortality and morbidity during the July 2006 heat wave in Porto, Portugal. Int J Biometeorol, 57(1), 155-167. https://doi.org/10.1007/s00484-012-0543-9

Montero, J. C., Miron, I. J., Criado, J. J., Linares, C., Diaz, J. (2010). Comparison between two methods of defining heat waves: a retrospective study in Castile-La Mancha (Spain). Sci Total Environ, 408(7), 1544-50. https://doi.org/10.1016/j.scitotenv.2010.01.013

Nair, H., Nokes, D. J., Gessner, B. D., Dherani, M., Madhi, S. A., Singleton, R. J., O'Brien, K. L., Roca, A., Wright, P. F., Bruce, N., Chandran, A., Theodoratou, E., Sutanto, A., Sedyaningsih, E. R., Ngama, M., Munywoki, P. K., Kartasasmita, C., Simões, E. A. F., Rudan, I., Weber, M. W., Campbell, H. (2010). Global burden of acute lower respiratory infections due to respiratory syncytial virus in young children: a systematic review and metaanalysis. The Lancet, 375(9725), 1545-1555. https://doi.org/10.1016/S01406736(10)60206-1

Numminen, E., Chewapreecha, C., Turner, C., Goldblatt, D., Nosten, F., Bentley, S. D., Turner, P., Corander, J. (2015). Climate induces seasonality in pneumococcal transmission. Scientific Reports, 5, 11344. https://doi.org/10.1038/srep11344 
Oluleye, A., Akinbobola, A. (2010). Malaria and pneumonia occurrence in Lagos, Nigeria: role of temperature and rainfall. African Journal of Environmental Science and Technology, 4(8), 506-16. Consultado em: https://www.ajol.info/index.php/ajest/article/view/71305

O'Neill, M. S., Hajat, S., Zanobetti, A., Ramirez-Aguilar, M., Schwartz, J. (2005). Impact of control for air pollution and respiratory epidemics on the estimated associations of temperature and daily mortality. Int $J$ Biometeorol, 50(2), 121-129. https://doi.org/10.1007/s00484-005-0269-z

Peixoto, A. (1975). Clima e saúde. São Paulo: Cia Editora Nacional, Brasiliana, 2 ed., v. 129.

Pessôa, C. L. C., Pessôa, R. S. (2009). Epidemiologia da DPOC no presente - aspectos nacionais e internacionais. Pulmão, 1, 7-12. Consultado em: http://www.sopterj.com.br/wpcontent/themes/ sopterj redesign 2017/ revista/atualizacao tematica/01.pdf

PORDATA - Base de dados Portugal contemporâneo s/d. População. Fundação Francisco Manuel dos Santos. Consultado em: https://www.pordata.pt/DB/Ambiente+de+Consulta/Nova+Consulta

Rogers, L. (1925). Relationship between pneumonia incidence and climate in India. The Lancet, 205(5310), 1173-1177. https://doi.org/10.1016/S0140-6736(01)24111-7

Rogerson, P. A. (2012). Métodos estatísticos para geografia: um guia para o estudante. Porto Alegre, $3^{\mathrm{a}}$ ed.: Bookman.

Sarfaty, M., Abouzaid, S. (2009). The physician's response to climate change. Family Medicine, 41(5), 358-63. Consultado em: https://www.ncbi.nlm.nih.gov/pubmed/19418286

Seo, J-H., Lee, J-Y. (2019). Novel nomogram based on risk factors of chronic obstructive pulmonary disease (COPD) using a naïve Bayesian classifier model. Journal of the Korean Statistical Society, 48(2), 278-86. https://doi.org/10.1016/j.jkss.2018.11.006

Silveira, R. B., Mendonça, M., Franke, A. E., Bitencourt, D. P. (2018). Impactos das ondas de frio sobre a saúde pública no município de São Joaquim - Santa Catarina - Brasil. Revista Brasileira de Climatologia, 22, 249-266. http://dx.doi.org/10.5380/abclima.v22i0.57776

Soebiyanto, R. P., Adimi, F., Kiang, R. K. (2010). Modeling and predicting seasonal influenza transmission in warm regions using climatological parameters. PLos ONE, 5(3), e9450. https://doi.org/10.1371/journal.pone.0009450

Sorre, M. (19519. Les Fondements de la Géographie Humaine (Les Fondements Biologiques: essai d'une écologie de l'homme). Paris: Librairie Armand Colin, Tome Premier.

Theoharatos, G., Pantavou, K., Mavrakis, A., Spanou, A., Katavoutas, G., Efstathiou, P., Mpekas, P., Asimakopoulos, D. (2010). Heat waves observed in 2007 in Athens, Greece: synoptic conditions, bioclimatological assessment, air quality levels and health effects. Environmental Research, 110(2), 152-161. https://doi.org/10.1016/j.envres.2009.12.002

Van Noort, S. P., Aguas, R., Ballesteros, S., Gomes, M. G. (2012). The role of weather on the relation between influenza and influenza-like illness. J Theor Biol, 298, 131-137. https://doi.org/10.1016/j.jtbi.2011.12.020

Viveiros, J. A. G. (2014). A influência das alterações climáticas nas patologias respiratórias. Dissertação em Medicina, Universidade de Coimbra. 74. Consultado em: https://estudogeral.sib.uc.pt/handle/10316/29245?mode=full 
Wang, L., Green, F. H. Y., Smiley-Jewell, S. M., Pinkerton, K. E. (2010). Susceptibility of the aging lung to environmental injury. Semin Respir Crit Care Med, 31(5), 539-53. https://doi.org/10.1055/s-0030-1265895

WHO - World Health Organization (2008). World Health Statistics. WHO Library, France. Consultado em: https://www.who.int/whosis/whostat/EN WHS08 Full.pdf?ua=1

WHO - World Health Organization (2018). Global tuberculosis report 2018. Geneva: World Health Organization (WHO). Consultado em: https://www.who.int/tb/publications/global report/en/

Wichmann, J., Andersen, Z., Ketzel, M., Ellermann, T., Loft, S. (2011). Apparent temperature and cause-specific emergency hospital admissions in Greater Copenhagen, Denmark. PLoS ONE, 6(7), e22904. https://doi.org/10.1371/journal.pone.0022904

WMO - World Meteorological Organization (2015). Guidelines on the definition and monitoring of extreme weather and climate events: draft version - first review by TT-DEWCE. Consultado em: https://public.wmo.int/en

Yusuf, S., Piedimonte, G., Auais, A., Demmler, G., Krishnan, S., Van Caeseele, P., Singleton, R., Broor, S., Parveen, S., Avendano, L., Parra, J., Chavez-Bueno, S., Murguía de Sierra, T., Simões, E. A. F., Shaha, S., Welliver, R. (2007). The relationship of meteorological conditions to the epidemic activity of respiratory syncytial virus. Epidemiology and Infection, 135(7), 107710-90. https://doi.org/10.1017/S095026880600776X

Zhao, Q., Li, S., Coelho, M. S. Z. S., Saldiva, P. H. N., Hu, K., Huxley, R. R., Abramson, M. J., Guo, Y. (2019). The association between heatwaves and risk of hospitalization in Brazil: a nationwide time series study between 2000 and 2015. PLoS Med, 16(2), e1002753-e. https://doi.org/10.1371/journal.pmed.1002753

Artigo recebido em / Received on: 23/07/2019

Artigo aceite para publicação em / Accepted for publication on: 20/10/2019 